medRxiv preprint doi: https://doi.org/10.1101/2021.01.04.21249208; this version posted January 5, 2021. The copyright holder for this preprint (which was not certified by peer review) is the author/funder, who has granted medRxiv a license to display the preprint in perpetuity.

It is made available under a CC-BY 4.0 International license .

\title{
Triglyceride Glucose Index Predicts Risk of Adverse Cardio-metabolic and Mortality Outcomes Among Chinese Adults: A Territory-Wide Longitudinal Study
}

\author{
Jiandong Zhou $*^{1}$, Sharen Lee $*^{2}$, Jeremy Hui ${ }^{3}$, Wing Tak Wong $\mathrm{PhD}^{4}$, Keith SK Leung BSc ${ }^{5}$, \\ Abraham KC Wai MBChB FRCP FRCEM ${ }^{5}$, Tong Liu MD PhD ${ }^{6}$, Bernard Man Yung Cheung PhD \\ FRCP $^{7}$, Gary Tse PhD FRCP \# ${ }^{6}$, Qingpeng Zhang PhD \# ${ }^{1}$ \\ ${ }^{1}$ School of Data Science, City University of Hong Kong, Hong Kong, Hong Kong, China \\ ${ }^{2}$ Cardiovascular Analytics Group, Laboratory of Cardiovascular Physiology, Hong Kong, China \\ ${ }^{5}$ Li Ka Shing Faculty of Medicine, The University of Hong Kong, Hong Kong, China \\ ${ }^{4}$ School of Life Sciences, The Chinese University of Hong Kong, Hong Kong, China \\ ${ }^{5}$ Emergency Medicine Unit, Li Ka Shing Faculty of Medicine, The University of Hong Kong, Hong \\ Kong, China \\ ${ }^{6}$ Tianjin Key Laboratory of Ionic-Molecular Function of Cardiovascular Disease, Department of \\ Cardiology, Tianjin Institute of Cardiology, Second Hospital of Tianjin Medical University, Tianjin \\ 300211, China \\ ${ }^{7}$ Division of Clinical Pharmacology and Therapeutics, Department of Medicine, The University of \\ Hong Kong, Pokfulam, Hong Kong, China
}

\# Correspondence to:

Prof. Gary Tse PhD FRCP

Tianjin Key Laboratory of Ionic-Molecular Function of Cardiovascular Disease, Department of Cardiology, Tianjin Institute of Cardiology, Second Hospital of Tianjin Medical University, Tianjin 300211, China

Faculty of Health and Medical Sciences, University of Surrey, GU2 7AL, Guildford, United Kingdom

Email: gary.tse@doctors.org.uk

Prof. Qingpeng Zhang PhD

School of Data Science, City University of Hong Kong,

Hong Kong, China

Email: qingpeng.zhang@ cityu.edu.hk 
medRxiv preprint doi: https://doi.org/10.1101/2021.01.04.21249208; this version posted January 5, 2021. The copyright holder for this preprint (which was not certified by peer review) is the author/funder, who has granted medRxiv a license to display the preprint in perpetuity.

It is made available under a CC-BY 4.0 International license.

\begin{abstract}
Background: The triglyceride glucose (TyG) index has been proposed to be a surrogate of insulin resistance. In the study, we aimed to examine the relationship between TyG index and the risk of new onset DM and secondary outcomes included atrial fibrillation (AF), heart failure (HF), acute myocardial infarction (AMI), ventricular tachycardia/fibrillation (VTF), cardiovascular mortality (CAD) and all-cause mortality.
\end{abstract}

Methods: The retrospective observational study analyzed patients recruited from $1^{\text {st }}$ January 2000 to $31^{\text {st }}$ December 2003 and followed up until $31^{\text {st }}$ December 2019. Demographics, past comorbidities, medications and laboratory tests were extracted. At baseline and follow-up, DM was defined as 1) any occasion $\mathrm{Hb} 1 \mathrm{Ac} \geq 14 \mathrm{~g} / \mathrm{dL}, 2)$ fasting glucose $\geq 7 \mathrm{mmol} / \mathrm{L}$ on two occasions, or 3) with DM diagnosis. We excluded 1) patients with prior DM or with the use of antidiabetic medications; 2) patients with prior $\mathrm{AMI} / \mathrm{HF} / \mathrm{AF}$ or with the use of diuretic/beta blockers for HF were excluded. Univariate analysis and multivariate Cox analysis with adjustments on demographics, past comorbidities and medications were conducted to identify the significant risk predictors of primary and secondary outcomes. Optimal cutoffs of TyG index for the primary and secondary outcomes were found with maximally selected rank statistics approach.

Result: Lager TyG index is significantly associated with new onset DM (HR: 1.51, 95\% CI: [1.47, 1.55], P vlaue<0.0001), new onset HF (HR: 1.27, 95\% CI: [1.2, 1.34], P vlaue<0.0001), new onset AF (HR: 2.36, 95\% CI: [2.26, 2.46], P vlaue<0.0001), new onset AMI (HR: 1.51, 95\% CI: [1.42, 1.6], P vlaue<0.0001), new onset VTF (HR: 1.22, 95\% CI: [1.13, 1.31], P vlaue<0.0001), new onset CAD (HR: 1.56, 95\% CI: [1.45, 1.69], P value<0.0001) and all-cause mortality (HR: 1.21, 95\% CI: $[1.18,1.25]$, P vlaue $<0.0001)$. TyG index and its 3rd tertile remained significant after being adjusted 
medRxiv preprint doi: https://doi.org/10.1101/2021.01.04.21249208; this version posted January 5, 2021. The copyright holder for this preprint (which was not certified by peer review) is the author/funder, who has granted medRxiv a license to display the preprint in perpetuity.

It is made available under a CC-BY 4.0 International license .

with significant demographics, past comorbidities and medicactions in multivariate cox models $(\mathrm{HR}>1, \mathrm{P}$ value<0.05). Optimal cut-off values of baseline TyG index and adjusted multivariate restricted cubic spline models further uncovered detailed associations of larger baseline TyG index with the primary and secondary outcome.

\section{Conclusion:}

Higher TyG index remained significantly associated with the elevated risk of new onset DM, AF, HF, AMI, VTF, CAD and all-cause mortality after adjustments on demographics, past comorbidities, and medications. 
medRxiv preprint doi: https://doi.org/10.1101/2021.01.04.21249208; this version posted January 5, 2021. The copyright holder for this preprint (which was not certified by peer review) is the author/funder, who has granted medRxiv a license to display the preprint in perpetuity.

It is made available under a CC-BY 4.0 International license .

\section{Introduction}

The triglyceride glucose (TyG) index has been proposed to be a surrogate of insulin resistance. It has been shown to predict adverse cardiovascular events in patients with diabetes alone, or with acute coronary syndrome (1), poor prognosis in ischaemic stroke patients (2) and incident diabetes in the general population (3). In the study, we aimed to examine the relationship between TyG index and new onset DM, atrial fibrillation (AF), heart failure (HF), acute myocardial infarction (AMI), ventricular tachycardia/fibrillation (VTF), cardiovascular mortality (CAD) and all-cause mortality.

\section{Methods}

\section{Study Population}

The crude inclusion criteria were as follows: 29944 patients with complete triglyceride and fast glucose tests were recruited from January $1^{\text {st }}, 2000$ to December $31^{\text {st }}, 2003$ and were followed up until Dec 31 ${ }^{\text {st }}, 2019$ (Figure 1). In total 5595 patients being excluded with the criteria were as follows: 1) with baseline DM diagnosis ( $\mathrm{N}=1769)$; 2) With any prior HbA1c occasion $\geq 14 \mathrm{gd} / \mathrm{L}$ ( $\mathrm{N}=1195)$; 3) With any two occasions of fast glucose $\geq 7 \mathrm{mmol} / \mathrm{L}(\mathrm{N}=1037)$; 4) With prior use of antidiabetic medications ( $\mathrm{N}=971)$; 5) With prior AMI $(\mathrm{N}=31)$; 6) With prior $\mathrm{HF}(\mathrm{N}=54)$; 7) With prior AF (N=105); 8) With prior HF (or use of diuretics/beta blockers for HF) (N=434) before the initial date of fast glucose or triglyceride tests (baseline). A total of 24349 patients fulfilled the eligibility criteria and were included in the study cohort.

Clinical and biochemical data were extracted for the present study. Patients' demographics include male gender and baseline age. Prior comorbidities were extracted, including liver diseases, endocrine, hypertension, ischemic heart disease (IHD), chronic obstructive pulmonary disease (COPD), gastrointestinal, peripheral vascular disease (PVD), transient ischemic attack 
medRxiv preprint doi: https://doi.org/10.1101/2021.01.04.21249208; this version posted January 5, 2021. The copyright holder for this preprint (which was not certified by peer review) is the author/funder, who has granted medRxiv a license to display the preprint in perpetuity.

It is made available under a CC-BY 4.0 International license .

(TIA)/ischemic stroke, gastrointestinal bleeding, and cancer. Standard Charlson comorbidity index was also calculated. Mortality was recorded using the International Classification of Diseases Tenth Edition (ICD-10) coding, whilst the outcome of DM diagnosis and comorbidities were documented in CDARS under ICD-9 codes. ICD-10 codes I00-I78 were used to identify cardiovascular mortality. Supplementary Table 1 displays the ICD-9 codes used to search for patient comorbidities.

Medications histories were also extracted, including angiotensinogen converting enzyme inhibitor (ACEI), angiotensin receptor blocker (ARB), calcium channel blockers, beta blockers, diuretics for $\mathrm{HF}$, diuretics for hypertension, nitrates, antihypertensive drugs, anti-diabetic drugs, statins and fibrates, lipid-lowering drugs, anticoagulants, and antiplatelets. In addition, baseline biochemical data, defined as complete cell count, renal and liver function tests, and glycemic and lipid profile measured from January 1st, 2000 to December 31st, 2003 were extracted. Complete cell count include mean corpuscular volume (MCV), lymphocyte, metamyelocyte, monocyte, neutrophil, white cell count, mean corpuscular hemoglobin $(\mathrm{MCH})$, myelocyte, platelet, reticulocyte, red cell count, and hematocrit (HCT). Liver and renal function tests include potassium, albumin, sodium, urea, protein, creatinine, alkaline phosphatase (ALP), aspartate transaminase, alanine aminotransferase (ALT), and bilirubin. Lipid and glycemic profile tests include low-density lipoprotein, high-density lipoprotein, HbA1c, cholesterol, fast glucose, and triglycerides. The TyG index was calculated as the $\ln (18 \times$ triglyceride level $[\mathrm{mmol} / \mathrm{L}] \times 18 \times$ fast glucose level $[\mathrm{mmol} / \mathrm{L}] / 2)$. Deciles and tertiles of TyG index were calculated.

Primary outcomes and statistical Analysis 
medRxiv preprint doi: https://doi.org/10.1101/2021.01.04.21249208; this version posted January 5, 2021. The copyright holder for this preprint (which was not certified by peer review) is the author/funder, who has granted medRxiv a license to display the preprint in perpetuity.

It is made available under a CC-BY 4.0 International license.

The primary outcome was new onset DM and the secondary outcomes included new onset $\mathrm{AF} / \mathrm{HF} / \mathrm{AMI} / \mathrm{VTF} / \mathrm{CAD}$ and all-cause mortality. The endpoint date of interest for all eligible patients was event presentation date, mortality, or study end (December 31, 2019). There are no records with follow-up loss. Descriptive statistics were used to summarize patients' characteristics of the primary outcome and secondary outcomes. Continuous variables were presented as median (95\% confidence interval $[\mathrm{CI}]$ or interquartile range $[\mathrm{IQR}])$ and categorical variables were presented as count (\%). The Mann-Whitney U test was used to compare two continuous variables. The Kruskal-Wallis test was used to determine whether more than two continuous variables have a different distribution. The $\chi 2$ test with Yates' correction was used for $2 \times 2$ contingency data. The study cohort was compared according to the tertile subgroups of TyG index (Table 1) and those with/without event presentation (Supplementary Tables 2-4).

Univariate Cox hazard proportional analysis models were used to identify the significant risk predictors of primary and secondary outcomes. Hazard ratios (HRs) with corresponding 95\% CIs and $\mathrm{P}$ values were reported accordingly. Kaplan-Meier curves were plotted against the time-to-event for primary and secondary outcomes stratified by deciles of TyG index (Figure 2). Adjusted cubic spline models of the associations between TyG index and the risk of primary and secondary outcomes were presented (Figure 3). Hazard ratios (HRs) of primary and secondary outcomes according to tertiles of TyG index were summarized (Table 4). Optimal cut-offs of baseline TyG index for primary and secondary outcomes were derived with maximal survival rank statistics approach after adjusting for significant demographics, past comorbidities, and medications with multivariate Cox model, and compared with $1^{\text {st }}$ tertile subgroup as reference (Table 5). Adjusted cubic spline model of the associations between TyG index and risk of new-onset DM and all-cause 
medRxiv preprint doi: https://doi.org/10.1101/2021.01.04.21249208; this version posted January 5, 2021. The copyright holder for this preprint (which was not certified by peer review) is the author/funder, who has granted medRxiv a license to display the preprint in perpetuity.

It is made available under a CC-BY 4.0 International license.

mortality were presented (Figure 4). All significance tests were two-tailed and considered statistically significant if $\mathrm{P}$ values were 0.05 . Data analyses were performed using RStudio software (Version: 1.1.456) and Python (Version: 3.6).

\section{Results}

\section{Clinical and Biochemical Characteristics}

The present cohort consists of 24349 patients (40.2\% males, median age of initial triglyceride test: 62.5 years old, IQR: 51.3-71.4, max: 100.97 years old). In total 9103 patients (37.38\%) passed away due to all-cause mortality after a median follow-up of 6451 days (IQR: 5142-6911, max: 7517 days), among them 1113 patients (4.57\%) died from cardiovascular diseases. Overall 8324 patients (34.18\%) developed new onset DM after a median follow up of 6149 days (IQR: 1854-6727, max: 7479 days), 2404 patients (9.87\%) developed new onset HF after a median follow up of 6431 days (IQR: 4871-6902, max: 7517 days), 2855 patients (11.72\%) developed new onset AF after a median follow up of 6399 days (IQR: 4567-6887, max: 7517 days), 1862 patients (7.64\%) developed new onset AMI after a median follow up of 6420 days (IQR: 4808-6895, max: 7517 days), 1275 patients (5.23\%) developed new onset VTF after a median follow up of 6445 days (IQR: 5049-6908, max: 7517 days).

The crude incidence rates were $33.11 \%(\mathrm{~N}=8062), 33.53 \%(\mathrm{~N}=8166)$ and $33.35 \%(\mathrm{~N}=8121)$ for baseline TyG index in the 1st, 2nd, and 3rd tertiles, respectively, as shown in Table $\mathbf{1}$ for the baseline characteristics according to the tertile subgroups of baseline TyG index. There exist significant differences among the three tertiles of TyG index in incidence rates of all-cause mortality (32.69\% v.s. $37.68 \%$ v.s. $41.74 \%$, P value<0.0001), CAD $(3.15 \%$ v.s. $4.38 \%$ v.s. $6.16 \%$, P 
medRxiv preprint doi: https://doi.org/10.1101/2021.01.04.21249208; this version posted January 5, 2021. The copyright holder for this preprint (which was not certified by peer review) is the author/funder, who has granted medRxiv a license to display the preprint in perpetuity.

It is made available under a CC-BY 4.0 International license.

value<0.0001), new DM (24.64\% v.s. $33.85 \%$ v.s. $43.98 \%$, P value<0.0001), new onset HF (7.98\% v.s. $10.39 \%$ v.s. $11.21 \%$, $\mathrm{P}$ value $<0.0001)$, new onset $\mathrm{AF}(3.80 \%$ v.s. $9.57 \%$ v.s. $21.74 \%$, $\mathrm{P}$ value $<0.0001)$, new onset AMI (5.49\% v.s. $7.17 \%$ v.s. $10.25 \%$, P value<0.0001) and new onset VTF (4.50\% v.s. $5.31 \%$ v.s. $5.88 \%$, P value $=0.0008)$.

Significant differences among the three tertile subgroups of patients were also observed in male gender $(37.78 \%$ v.s. $40.87 \%$ v.s. $41.92 \%$, P value=0.0012), baseline age (median: 60.49 years old, IQR: 49.33-70.94 years old; max: 100.97 years old v.s. median: 63.05 years old, IQR: 52.14-71.81; max: 96.23 years old v.s. median: 63.48 years old, IQR: 52.14-71.44; max: 98.94 years old, P value<0.0001), Charlson score (median: 2.0, IQR: 0.0-3.0; max: 11.0 v.s. median: 2.0, IQR: 1.0-3.0; max: 9.0 v.s. median: 2.0, IQR: 1.0-3.0; max: 9.0, P value<0.0001), COPD (0.23\% v.s. 0.07\% v.s. $0.07 \%$, $\mathrm{P}$ value $=0.0039)$, and medication use of ACEI $(5.43 \%$ v.s. $7.43 \%$ v.s. $9.59 \%$, $\mathrm{P}$ value $<0.0001)$, calcium channel blockers $(9.36 \%$ v.s. $11.84 \%$ v.s. $12.88 \%$, P value $<0.0001)$, beta blockers $(8.71 \%$ v.s. $11.36 \%$ v.s. $12.17 \%$, $\mathrm{P}$ value<0.0001), nitrates $(3.88 \%$ v.s. $4.65 \%$ v.s. $5.27 \%$, $\mathrm{P}$ value $=0.0003)$, anti-diabetic drugs $(2.70 \%$ v.s. $4.83 \%$ v.s. $8.81 \%$, P value $<0.0001)$, statins and fibrates $(5.80 \%$ v.s. $7.46 \%$ v.s. $9.77 \%$, P value<0.0001), diuretics for hypertension $(4.44 \%$ v.s. $6.00 \%$ v.s. $6.09 \%$, $\mathrm{P}$ value $<0.0001)$, anticoagulants $(0.11 \%$ v.s. $0.22 \%$ v.s. $0.34 \%$, $\mathrm{P}$ value $=0.0077)$, antiplatelets $(4.70 \%$ v.s. $5.55 \%$ v.s. $6.44 \%$, $\mathrm{P}$ value $<0.0001)$ and lipid-lowering drugs $(4.66 \%$ v.s. $6.38 \%$ v.s. $7.38 \%$, $\mathrm{P}$ value $<0.0001)$.

Patients in the $3^{\text {rd }}$ tertile subgroup had significantly lower levels of mean corpuscular volume, mean corpuscular haemoglobin, platelet, reticulocyte, red cell count, hematocrit, urate, albumin, sodium, urea, protein, creatinine, bilirubin and high-density lipoprotein, while had higher levels of 
medRxiv preprint doi: https://doi.org/10.1101/2021.01.04.21249208; this version posted January 5, 2021. The copyright holder for this preprint (which was not certified by peer review) is the author/funder, who has granted medRxiv a license to display the preprint in perpetuity.

It is made available under a CC-BY 4.0 International license .

lymphocyte, monocyte, neutrophil, white cell count, ALP, aspartate transaminase, ALT, triglyceride, low-density lipoprotein, cholesterol, $\mathrm{HbA1c}$ and fast glucose $(\mathrm{P}$ value $<0.05)$.

In addition, the baseline characteristics of patients with/without event presentation of new onset DM/AF/HF/AMI/VTF/CAD and all-cause mortality were presented in Supplementary Tables 2-4. Patients in the third tertile had a significant larger value of TyG index (median: 6.7, IQR: 6.45-6.88; max: 7.03 v.s. median: 7.31, IQR: 7.17-7.46; max: 7.63 v.s. median: 8.04, IQR: 7.17-8.4; max: 11.61, $\mathrm{P}$ value $<0.0001)$. Kaplan-Meier survival curves of the primary and secondary outcomes stratified by deciles of baseline TyG index were presented in Figure 2.

\section{Significant unirivate risk factors of primary and secondary outcomes}

Cox-proportional hazard model analysis identified the significant risk factors of primary and secondary outcomes as shown in Table 2 and Table 3. Important predictors of new onset DM include male gender, older patient age, larger Charlson score, comorbidities of liver diseases, IHD, COPD, and PVD (HR>1, P value<0.05); higher levels of mean corpuscular volume, monocyte, neutrophil, white cell count, mean corpuscular haemoglobin, platelet, reticulocyte, hematocrit, potassium, urea, creatinine, alkaline phosphatase, aspartate transaminase, alanine transaminase, bilirubin, triglyceride, $\mathrm{HbA1c}$, fast glucose $(\mathrm{HR}>1, \mathrm{P}$ value $<0.05)$, and lower levels of lymphocyte, albumin, sodium, protein, low-density lipoprotein, high-density lipoprotein, and cholesterol (HR<1, $\mathrm{P}$ value<0.05). Lager TyG index is significantly associated with new onset DM (HR: $1.51,95 \%$ CI: $[1.47,1.55]$, P vlaue<0.0001). Significances of the stratified decciles and tertiles of TyG index with new onset DM were also presented in Table 2. Medication prescriptions of all the mentioned drugs were associated with new onset $\mathrm{DM}(\mathrm{HR}>1, \mathrm{P}$ value $<0.05)$. 
medRxiv preprint doi: https://doi.org/10.1101/2021.01.04.21249208; this version posted January 5, 2021. The copyright holder for this preprint (which was not certified by peer review) is the author/funder, who has granted medRxiv a license to display the preprint in perpetuity.

It is made available under a CC-BY 4.0 International license .

Significant risk predictors of new onset HF include male gender, older patient age, larger Charlson score, comorbidities of hypertension, IHD, COPD, PVD, TIA/Ischemic stroke and cancer $(\mathrm{HR}>1$, $\mathrm{P}$ value $<0.05)$; higher levels of mean corpuscular volume, monocyte, neutrophil, white cell count, mean corpuscular haemoglobin, platelet, urea, creatinine, alkaline phosphatase, HbA1c, fast glucose $(\mathrm{HR}>1, \mathrm{P}$ value $<0.05)$, and lower levels of lymphocyte, red cell count, albumin, sodium, protein, alanine transaminase, high-density lipoprotein, and cholesterol $(\mathrm{HR}<1, \mathrm{P}$ value $<0.05)$. Lager TyG index is significantly associated with new onset HF (HR: 1.27, 95\% CI: [1.2, 1.34], P vlaue $<0.0001)$. Significances of the stratified decciles and tertiles of TyG index with new onset DM were also presented. Medication prescriptions of all the mentioned drugs were associated with new onset $\mathrm{HF}(\mathrm{HR}>1, \mathrm{P}$ value $<0.05)$.

Significant predictors of new onset AF included older patient age, larger Chalson score, past comorbidities of hypertension, IHD, COPD, and gastrointestinal bleeding $(\mathrm{HR}<1, \mathrm{P}$ value $<0.05)$; higher levels of mean corpuscular volume, monocyte, neutrophil, white cell count, mean corpuscular haemoglobin, urea, creatinine, alkaline phosphatase, bilirubin, triglyceride, HbA1c, fast glucose $(\mathrm{HR}>1, \mathrm{P}$ value $<0.05)$, and lower levels of lymphocyte, platelet, red cell count, potassium, albumin, protein, low-density lipoprotein, and cholesterol $(\mathrm{HR}<1, \mathrm{P}$ value $<0.05)$. Lager TyG index is significantly associated with new onset AF (HR: 2.36, 95\% CI: [2.26, 2.46], P vlaue<0.0001). Significances of the stratified decciles and tertiles of TyG index with new onset AF were also presented. Medication prescriptions of ACEI, ARB, calcium channel blockers, beta blockers, nitrates, antihypertensive drugs, diuretics for hypertension, anticoagulants and antiplatelets were associated with new onset $\mathrm{AF}(\mathrm{HR}>1, \mathrm{P}$ value $<0.05)$. 
medRxiv preprint doi: https://doi.org/10.1101/2021.01.04.21249208; this version posted January 5, 2021. The copyright holder for this preprint (which was not certified by peer review) is the author/funder, who has granted medRxiv a license to display the preprint in perpetuity.

It is made available under a CC-BY 4.0 International license.

Significant univariate risk predictors of new onset AMI included male gender, older baseline age, larger Charlson score, past comorbidities of hypertension, IHD, COPD, gastrointestinal diseases, PVD, TIA/Ischemic stroke $(\mathrm{HR}>1, \mathrm{P}$ value $<0.05)$; higher levels of mean corpuscular volume, monocyte, neutrophil, white cell count, mean corpuscular haemoglobin, platelet, reticulocyte, urea, creatinine, alkaline phosphatase, HbA1c, fast glucose $(\mathrm{HR}>1, \mathrm{P}$ value $<0.05)$, and lower levels of lymphocyte, platelet, red cell count, sodium, protein and high-density lipoprotein $(\mathrm{HR}<1, \mathrm{P}$ value $<0.05$ ). Lager TyG index is significantly associated with new onset AMI (HR: 1.51, 95\% CI: $[1.42,1.6]$, P vlaue $<0.0001)$. Significances of the stratified decciles and tertiles of TyG index with new onset AMI were also presented. Medication prescriptions of all the mentioned drugs were associated with new onset AMI $(\mathrm{HR}>1, \mathrm{P}$ value $<0.05)$.

Signifiant risk predictors of new osnet VTF included male gender, older patient age, larger Charlson score, past comorbidities of hypertension, IHD, PVD, TIA/Ischemic stroke (HR>1, P value<0.05); higher levels of mean corpuscular volume, neutrophil, white cell count, mean corpuscular haemoglobin, urea, creatinine, HbA1c, fast glucose $(\mathrm{HR}>1, \mathrm{P}$ value $<0.05)$, and lower levels of lymphocyte, platelet, red cell count, sodium, protein and high-density lipoprotein $(\mathrm{HR}<1, \mathrm{P}$ value $<0.05)$. Lager TyG index is significantly associated with new onset VTF (HR: 1.22, 95\% CI: $[1.13,1.31]$, P vlaue $<0.0001)$. Significances of the stratified decciles and tertiles of TyG index with new onset VTF were also presented. Medication prescriptions of all the mentioned drugs were associated with new onset $\mathrm{VTF}(\mathrm{HR}>1, \mathrm{P}$ value $<0.05)$.

Adjusted associations of baseline TyG index with primary and secondary outcomes 
medRxiv preprint doi: https://doi.org/10.1101/2021.01.04.21249208; this version posted January 5, 2021. The copyright holder for this preprint (which was not certified by peer review) is the author/funder, who has granted medRxiv a license to display the preprint in perpetuity.

It is made available under a CC-BY 4.0 International license .

With $1^{\text {st }}$ tertile subgroups of baseline TyG index as a reference, it has been found that the $3^{\text {rd }}$ tertile subgroup were more likely to developed new onset DM/AF/HF/AMI/VTF and meet the outcomes of $\mathrm{CAD}$ and all-cause mortality $(\mathrm{HR}>1, \mathrm{P}$ value $<0.05)$ (Table 4), after being adjusted for significant demographics, past comorbidities, and medications in different multivariate Cox analysis models. The optimal cut-off values of baseline TyG index with primary and secondary outcomes were identified with the maximal survival rank statistics approach (Table 5). Adjusted multivariate restricted cubic spline models identified the associations between baseline TyG index and HR of primary and secondary outcomes were presented in Figure 3.

It has been further confirmed that TyG index above the optimal cut-offs was associated with elevated adverse risk of both the primary and secondary outcomes. Analyses of the associations between baseline TyG index and primary and secondary outcomes with maximal survival rank statistics approach were presented in Figure 4. Subgroup characteristics of triglyceride cut-offs with new onset DM and all-cause mortality were presented in Supplementary Table 2 and Table 3, respectively. Basic characteristics of TyG index cut-offs with primary and secondary outcomes were compared in Supplementary Tables 5-7.

\section{Discussion}

The major findings of the present study are summarized as follows:

1). Lager TyG index is significantly associated with new onset DM (HR: 1.51, 95\% CI: [1.47, 1.55], P vlaue<0.0001), new onset HF (HR: 1.27, 95\% CI: [1.2, 1.34], P vlaue<0.0001), new onset AF (HR: 2.36, 95\% CI: [2.26, 2.46], P vlaue<0.0001), new onset AMI (HR: 1.51, 95\% CI: [1.42, 1.6], P vlaue<0.0001), new onset VTF (HR: 1.22, 95\% CI: [1.13, 1.31], P vlaue<0.0001), new onset 
CAD (HR: 1.56, 95\% CI: [1.45, 1.69], P value<0.0001) and all-cause mortality (HR: 1.21, 95\% CI:

$[1.18,1.25], \mathrm{P}$ vlaue $<0.0001)$

2). $3^{\text {rd }}$ tertile subgroup $(\mathrm{TyG}>7.63)$ were more likely to developed new onset $\mathrm{DM} / \mathrm{AF} / \mathrm{HF} / \mathrm{AMI} / \mathrm{VTF}$ and meet the outcomes of $\mathrm{CAD}$ and all-cause mortality (HR>1, $\mathrm{P}$ value $<0.05)$;

3). TyG index and its $3^{\text {rd }}$ tertile remained significant after being adjusted with significant demographics, past comorbidities and medicactions in multivariate cox models (HR>1, P value $<0.05)$;

4). Optimal cut-off values of baseline TyG index were identified and subgroups with TyG index larger than the cut-offs were significantly associated with the primary and secondary outcome (HR>1, $\mathrm{P}$ value $<0.05)$;

5). Adjusted multivariate restricted cubic spline models further uncovered the detailed associations between baseline TyG index and HRs of primary and secondary outcomes.

\section{Limitations}

As in other observational studies, this study is limited by potential under-coding of comorbidities, missing data, and coding errors. Additionally, the duration of the complications and the prescribed treatments were not accounted for, which could affect the interpretation of blood pressure value and variability measurements. In addition, this study is conducted based on a Hong Kong cohort, and it is 
medRxiv preprint doi: https://doi.org/10.1101/2021.01.04.21249208; this version posted January 5, 2021. The copyright holder for this preprint (which was not certified by peer review) is the author/funder, who has granted medRxiv a license to display the preprint in perpetuity.

It is made available under a CC-BY 4.0 International license .

expected that external validity through comparisons with studies from other countries reporting the association between blood pressure variability and adverse outcomes could be conducted for further confirmation. Finally, all patients were older Chinese people, caution should be made when interpreting our findings in younger individuals and other ethnic populations.

\section{Conclusion}

Higher TyG index remained significantly associated with the elevated risk of new onset DM, AF, HF, AMI, VTF, CAD and all-cause mortality after adjustments on demographics, past comorbidities, and medications.

\section{Funding}

None.

\section{References}

1. Wang L, Cong H-l, Zhang J-x, Hu Y-c, Wei A, Zhang Y-y, Yang H, Ren L-b, Qi W, Li W-y, Zhang $\mathrm{R}, \mathrm{Xu} \mathrm{J}$-h. Triglyceride-glucose index predicts adverse cardiovascular events in patients with diabetes and acute coronary syndrome. Cardiovascular Diabetology. 2020 2020/06/13;19(1):80.

2. Zhou Y, Pan Y, Yan H, Wang Y, Li Z, Zhao X, Li H, Meng X, Wang C, Liu L, Wang Y. Triglyceride Glucose Index and Prognosis of Patients With Ischemic Stroke. Front Neurol. 2020;11:456.

3. Chen C-1, Liu L, Lo K, Huang J-y, Yu Y-1, Huang Y-q, Feng Y-q. Association Between Triglyceride Glucose Index and Risk of New-Onset Diabetes Among Chinese Adults: Findings From the China Health and Retirement Longitudinal Study. Frontiers in Cardiovascular Medicine. [Original Research]. 2020 2020-November-27;7(287). 
Table 1. Baseline characteristics of patients according to tertile subgroups of TyG index.

$*$ for $\mathrm{p} \leq 0.05, * *$ for $\mathrm{p} \leq 0.01, * * *$ for $\mathrm{p} \leq 0.001$; \# indicates the difference among three tertile subgroups

\begin{tabular}{|c|c|c|c|c|c|}
\hline $\begin{array}{l}\text { Characteristic } \\
\mathrm{s}\end{array}$ & $\begin{array}{l}\text { Overall }(\mathrm{N}=24349) \\
\text { Median }(\mathrm{IQR}) ; \mathrm{Max} ; \mathrm{N} \text { or } \\
\text { Count }(\%)\end{array}$ & $\begin{array}{l}\text { Tertile1 } \\
\text { TyG }<6.98 \\
(\mathrm{~N}=8062) \text { Median } \\
\text { (IQR);Max; N or Count }(\%)\end{array}$ & $\begin{array}{l}\text { Tertile2 } \\
\text { TyG } \in[6.98,7.63] \\
(\mathrm{N}=8166) \text { Median } \\
(\mathrm{IQR}) ; \text { Max;N or } \operatorname{Count}(\%)\end{array}$ & $\begin{array}{l}\text { Tertile3 } \\
\text { TyG }>7.63 \\
\text { (N=8121) Median } \\
\text { (IQR);Max; N or Count }(\%)\end{array}$ & P value ${ }^{\#}$ \\
\hline \multicolumn{6}{|l|}{ Outcomes } \\
\hline $\begin{array}{l}\text { All-cause } \\
\text { mortality }\end{array}$ & $9103(37.38 \%)$ & $2636(32.69 \%)$ & $3077(37.68 \%)$ & $3390(41.74 \%)$ & $\begin{array}{l}<0.0001 * * \\
*\end{array}$ \\
\hline $\begin{array}{l}\text { Cardiovascular } \\
\text { mortality }\end{array}$ & $1113(4.57 \%)$ & $254(3.15 \%)$ & $358(4.38 \%)$ & $501(6.16 \%)$ & $\begin{array}{l}<0.0001 * * \\
*\end{array}$ \\
\hline New onset DM & $8324(34.18 \%)$ & $1987(24.64 \%)$ & $2765(33.85 \%)$ & $3572(43.98 \%)$ & $\begin{array}{l}<0.0001 * * \\
*\end{array}$ \\
\hline New onset HF & $2404(9.87 \%)$ & $644(7.98 \%)$ & $849(10.39 \%)$ & $911(11.21 \%)$ & $\begin{array}{l}<0.0001 * * \\
*\end{array}$ \\
\hline New onset AF & $2855(11.72 \%)$ & $307(3.80 \%)$ & $782(9.57 \%)$ & $1766(21.74 \%)$ & $\begin{array}{l}<0.0001 * * \\
*\end{array}$ \\
\hline $\begin{array}{l}\text { New onset } \\
\text { AMI }\end{array}$ & $1862(7.64 \%)$ & $443(5.49 \%)$ & $586(7.17 \%)$ & $833(10.25 \%)$ & $\begin{array}{l}<0.0001 * * \\
*\end{array}$ \\
\hline New onset VTF & $F 1275(5.23 \%)$ & $363(4.50 \%)$ & $434(5.31 \%)$ & $478(5.88 \%)$ & $0.0008 * *$ \\
\hline \multicolumn{6}{|l|}{ Demographics } \\
\hline Male gender & $9789(40.20 \%)$ & $3046(37.78 \%)$ & $3338(40.87 \%)$ & $3405(41.92 \%)$ & $0.0012 *$ \\
\hline $\begin{array}{l}\text { Baseline age, } \\
\text { year }\end{array}$ & $\begin{array}{l}62.51(51.31-71.37) ; 100.97 ; n=243 \\
49\end{array}$ & $\begin{array}{l}60.49(49.33-70.94) ; 100.97 ; n=8 \\
62\end{array}$ & $\begin{array}{l}63.05(52.14-71.81) ; 96.23 ; \mathrm{n}=81 \\
6\end{array}$ & $\begin{array}{l}63.48(52.71-71.44) ; 98.94 ; n=81 \\
21\end{array}$ & $\begin{array}{l}<0.0001 * * \\
*\end{array}$ \\
\hline \multicolumn{6}{|l|}{$\begin{array}{l}\text { Past } \\
\text { comorbidities }\end{array}$} \\
\hline Charlson score & $2.0(1.0-3.0) ; 11.0 ; \mathrm{n}=24349$ & $2.0(0.0-3.0) ; 11.0 ; \mathrm{n}=8062$ & $2.0(1.0-3.0) ; 9.0 ; \mathrm{n}=8166$ & $2.0(1.0-3.0) ; 9.0 ; \mathrm{n}=8121$ & $\begin{array}{l}<0.0001 * * \\
*\end{array}$ \\
\hline Liver diseases & $7(0.02 \%)$ & $3(0.03 \%)$ & $2(0.02 \%)$ & $2(0.02 \%)$ & 0.8606 \\
\hline Endocrine & $31(0.12 \%)$ & $10(0.12 \%)$ & $12(0.14 \%)$ & $9(0.11 \%)$ & 0.8077 \\
\hline
\end{tabular}




\begin{tabular}{|c|c|c|c|c|c|}
\hline Hypertension & $1042(4.27 \%)$ & $326(4.04 \%)$ & $380(4.65 \%)$ & $336(4.13 \%)$ & 0.1405 \\
\hline IHD & $241(0.98 \%)$ & $82(1.01 \%)$ & $77(0.94 \%)$ & $82(1.00 \%)$ & 0.8729 \\
\hline COPD & $31(0.12 \%)$ & $19(0.23 \%)$ & $6(0.07 \%)$ & $6(0.07 \%)$ & $0.0039 * *$ \\
\hline Gastrointestinal & 1586(2.40\%) & $219(2.71 \%)$ & $185(2.26 \%)$ & $182(2.24 \%)$ & 0.0956 \\
\hline PVD & $13(0.05 \%)$ & $3(0.03 \%)$ & $7(0.08 \%)$ & $3(0.03 \%)$ & 0.3006 \\
\hline $\begin{array}{l}\text { TIA/Ischemic } \\
\text { stroke }\end{array}$ & $98(0.40 \%)$ & $27(0.33 \%)$ & $39(0.47 \%)$ & $32(0.39 \%)$ & 0.3561 \\
\hline $\begin{array}{l}\text { Gastrointestinal } \\
\text { bleeding }\end{array}$ & $114(0.46 \%)$ & $35(0.43 \%)$ & $42(0.51 \%)$ & $37(0.45 \%)$ & 0.7425 \\
\hline Cancer & $161(0.66 \%)$ & $59(0.73 \%)$ & $49(0.60 \%)$ & $53(0.65 \%)$ & 0.5851 \\
\hline \multicolumn{6}{|l|}{ Medications } \\
\hline ACEI & $1824(7.49 \%)$ & $438(5.43 \%)$ & $607(7.43 \%)$ & $779(9.59 \%)$ & $\begin{array}{l}<0.0001 * * \\
*\end{array}$ \\
\hline ARB & $89(0.36 \%)$ & $21(0.26 \%)$ & $33(0.40 \%)$ & $35(0.43 \%)$ & 0.1567 \\
\hline $\begin{array}{l}\text { Calcium } \\
\text { channel } \\
\text { blockers }\end{array}$ & $2768(11.36 \%)$ & $755(9.36 \%)$ & $967(11.84 \%)$ & $1046(12.88 \%)$ & $\begin{array}{l}<0.0001 * * \\
*\end{array}$ \\
\hline Beta blockers & $2620(10.76 \%)$ & $703(8.71 \%)$ & $928(11.36 \%)$ & $989(12.17 \%)$ & $\begin{array}{l}<0.0001 * * \\
*\end{array}$ \\
\hline Nitrates & $1121(4.60 \%)$ & $313(3.88 \%)$ & $380(4.65 \%)$ & $428(5.27 \%)$ & $0.0003 * *$ \\
\hline $\begin{array}{l}\text { Antihypertensi } \\
\text { ve drugs }\end{array}$ & $1056(4.33 \%)$ & $329(4.08 \%)$ & $379(4.64 \%)$ & $348(4.28 \%)$ & 0.2363 \\
\hline $\begin{array}{l}\text { Anti-diabetic } \\
\text { drugs }\end{array}$ & $1329(5.45 \%)$ & $218(2.70 \%)$ & $395(4.83 \%)$ & $716(8.81 \%)$ & $\begin{array}{l}<0.0001 * * \\
*\end{array}$ \\
\hline $\begin{array}{l}\text { Statins and } \\
\text { fibrates }\end{array}$ & $1872(7.68 \%)$ & $468(5.80 \%)$ & $610(7.46 \%)$ & 794(9.77\%) & $\begin{array}{l}<0.0001 * * \\
*\end{array}$ \\
\hline $\begin{array}{l}\text { Diuretics for } \\
\text { hypertension }\end{array}$ & $1343(5.51 \%)$ & $358(4.44 \%)$ & $490(6.00 \%)$ & $495(6.09 \%)$ & $\begin{array}{l}<0.0001 * * \\
*\end{array}$ \\
\hline Anticoagulants & $55(0.22 \%)$ & $9(0.11 \%)$ & $18(0.22 \%)$ & $28(0.34 \%)$ & $0.0077 * *$ \\
\hline Antiplatelets & $1356(5.56 \%)$ & $379(4.70 \%)$ & $454(5.55 \%)$ & $523(6.44 \%)$ & $<0.0001 * *$ \\
\hline
\end{tabular}




\begin{tabular}{|c|c|c|c|c|c|}
\hline $\begin{array}{l}\text { Lipid-lowering } \\
\text { drugs }\end{array}$ & $1497(6.14 \%)$ & $376(4.66 \%)$ & $521(6.38 \%)$ & $600(7.38 \%)$ & $\begin{array}{l}<0.0001 * * \\
*\end{array}$ \\
\hline \multicolumn{6}{|l|}{$\begin{array}{l}\text { Complete cell } \\
\text { count }\end{array}$} \\
\hline $\begin{array}{l}\text { Mean } \\
\text { corpuscular } \\
\text { volume, fL }\end{array}$ & $89.7(86.3-92.6) ; 132.3 ; \mathrm{n}=9866$ & $90.3(86.9-93.1) ; 119.5 ; \mathrm{n}=3237$ & $89.7(86.6-92.6) ; 132.3 ; n=3282$ & $89.1(85.7-92.1) ; 108.0 ; \mathrm{n}=3347$ & $\begin{array}{l}<0.0001 * * \\
*\end{array}$ \\
\hline $\begin{array}{l}\text { Lymphocyte, } \\
\text { x10^9/L }\end{array}$ & $1.8(1.4-2.3) ; 85.28 ; n=5602$ & $1.7(1.3-2.2) ; 5.4 ; \mathrm{n}=1858$ & $1.9(1.4-2.3) ; 85.28 ; n=1825$ & $1.9(1.5-2.5) ; 5.5 ; \mathrm{n}=1919$ & $\begin{array}{l}<0.0001 * * \\
*\end{array}$ \\
\hline $\begin{array}{l}\text { Metamyelocyte } \\
\text { x } 10^{\wedge} 9 / \mathrm{L}\end{array}$ & $0.19(0.09-0.62) ; 2.0 ; \mathrm{n}=45$ & $0.12(0.04-0.4) ; 1.1 ; \mathrm{n}=11$ & $0.23(0.1-0.58) ; 1.47 ; \mathrm{n}=8$ & $0.2(0.12-0.62) ; 2.0 ; \mathrm{n}=26$ & 0.3784 \\
\hline $\begin{array}{l}\text { Monocyte, } \\
\times 10^{\wedge} 9 / \mathrm{L}\end{array}$ & $0.5(0.4-0.6) ; 3.22 ; \mathrm{n}=5592$ & $0.41(0.3-0.6) ; 3.18 ; n=1854$ & $0.5(0.4-0.6) ; 2.3 ; \mathrm{n}=1823$ & $0.5(0.4-0.66) ; 3.22 ; \mathrm{n}=1915$ & $\begin{array}{l}<0.0001 * * \\
*\end{array}$ \\
\hline $\begin{array}{l}\text { Neutrophil, } \\
\text { x10^9/L }\end{array}$ & $4.3(3.3-5.9) ; 39.48 ; n=5590$ & $3.9(3.0-5.5) ; 36.67 ; n=1852$ & $4.3(3.3-5.8) ; 39.48 ; n=1823$ & $4.5(3.6-6.3) ; 28.2 ; n=1915$ & $\begin{array}{l}<0.0001 * * \\
*\end{array}$ \\
\hline $\begin{array}{l}\text { White cell } \\
\text { count, } \times 10^{\wedge} 9 / \mathrm{L}\end{array}$ & 7.1(5.9-8.7);91.7;n=9924 & $6.5(5.42-8.03) ; 40.3 ; n=3250$ & 7.1(5.9-8.69);91.7;n=3304 & $7.61(6.3-9.39) ; 30.8 ; n=3370$ & $\begin{array}{l}<0.0001 * * \\
*\end{array}$ \\
\hline $\begin{array}{l}\text { Mean } \\
\text { corpuscular } \\
\text { haemoglobin, } \\
\text { pg }\end{array}$ & $30.5(29.3-31.7) ; 44.1 ; n=9866$ & $30.7(29.4-31.8) ; 41.5 ; n=3237$ & $30.5(29.3-31.6) ; 44.1 ; \mathrm{n}=3282$ & $30.5(29.2-31.6) ; 38.0 ; \mathrm{n}=3347$ & $0.0016 * *$ \\
\hline $\begin{array}{l}\text { Myelocyte, } \\
\times 10^{\wedge} 9 / \mathrm{L}\end{array}$ & $0.22(0.11-0.52) ; 2.87 ; \mathrm{n}=30$ & $0.18(0.11-0.52) ; 0.81 ; \mathrm{n}=8$ & $0.11(0.08-0.24) ; 0.94 ; \mathrm{n}=7$ & $0.34(0.14-0.8) ; 2.87 ; \mathrm{n}=15$ & 0.4122 \\
\hline Platelet, & $237.0(198.0-282.0) ; 1250.0 ; \mathrm{n}=992$ & $232.0(192.0-275.0) ; 957.0 ; n=325$ & $237.0(200.0-283.0) ; 1250.0 ; n=33$ & $3241.0(203.0-285.0) ; 952.0 ; n=33$ & $<0.0001 * *$ \\
\hline $\mathrm{x} 10^{\wedge} 9 / \mathrm{L}$ & 4 & 0 & 04 & 70 & $*$ \\
\hline $\begin{array}{l}\text { Reticulocyte, } \\
\times 10^{\wedge} 9 / \mathrm{L}\end{array}$ & $58.65(40.69-87.04) ; 324.0 ; n=368$ & $49.11(33.77-80.31) ; 165.9 ; \mathrm{n}=116$ & $52.64(37.53-80.9) ; 324.0 ; n=123$ & $\begin{array}{l}70.05(46.2-91.77) ; 307.58 ; n=12 \\
9\end{array}$ & $0.0009 * * *$ \\
\hline $\begin{array}{l}\text { Red cell count, } \\
\mathrm{x} 10^{\wedge} 12 / \mathrm{L}\end{array}$ & $4.43(4.11-4.8) ; 7.62 ; n=9863$ & $4.35(4.06-4.69) ; 7.37 ; n=3236$ & $4.45(4.12-4.8) ; 7.61 ; n=3280$ & $4.49(4.15-4.88) ; 7.62 ; \mathrm{n}=3347$ & $\begin{array}{l}<0.0001 * * \\
*\end{array}$ \\
\hline
\end{tabular}


Hematocrit,

$\mathrm{L} / \mathrm{L}$

$0.39(0.37-0.42) ; 0.551 ; \mathrm{n}=1368 \quad 0.39(0.36-0.42) ; 0.52 ; \mathrm{n}=446$

$0.4(0.37-0.42) ; 0.52 ; \mathrm{n}=457$

$0.4(0.37-0.43) ; 0.55 ; \mathrm{n}=465$

$0.0022 * *$

\section{Liver and renal}

function tests

Potassium,

$\mathrm{mmol} / \mathrm{L}$

$4.2(3.9-4.5) ; 13.3 ; \mathrm{n}=23612$

$4.2(3.9-4.5) ; 9.3 ; n=7724$

$4.2(3.9-4.5) ; 13.3 ; \mathrm{n}=7911$

$4.2(3.87-4.51) ; 8.1 ; n=7977$

0.3551

Albumin, g/L

42.0(39.9-44.0);58.0;n=13367

$42.0(39.2-44.0) ; 56.0 ; \mathrm{n}=4325$

42.0(40.0-44.0);58.0;n=4547

$42.0(40.0-44.0) ; 56.0 ; \mathrm{n}=4495$

$<0.0001 * *$ $*$

Sodium, $\quad 140.2(139.0-142.0) ; 168.0 ; \mathrm{n}=2367141.0(139.0-142.0) ; 152.0 ; \mathrm{n}=774141.0(139.0-142.0) ; 158.0 ; \mathrm{n}=793140.0(138.0-142.0) ; 168.0 ; \mathrm{n}=79<0.0001 * *$ $\mathrm{mmol} / \mathrm{L}$

7

$5.5(4.5-6.6) ; 47.3 ; n=7746$

Urea, $\mathrm{mmol} / \mathrm{L} \quad 5.5(4.6-6.7) ; 47.3 ; \mathrm{n}=23665$

Protein, g/L 75.0(71.4-78.0);109.0;n=13305

$74.0(71.0-77.0) ; 100.0 ; \mathrm{n}=4303$

2

$5.6(4.6-6.7) ; 34.8 ; \mathrm{n}=7926$

91

5.6(4.6-6.8);46.4;n=7993

$<0.0001 * *$ $*$

$<0.0001^{* *}$

$75.0(72.0-78.0) ; 109.0 ; \mathrm{n}=4524$

Creatinine, umol/L

83.0(72.0-98.0);1509.0;n=23711 81.0(70.0-95.0);629.0;n=7762

$84.0(72.0-98.0) ; 807.0 ; \mathrm{n}=7944$

Alkaline phosphatase,

$\mathrm{U} / \mathrm{L}$

Aspartate

transaminase,

$\mathrm{U} / \mathrm{L}$

Alanine

transaminase, $\quad 21.0(15.0-30.0) ; 918.0 ; \mathrm{n}=10416$

$\mathrm{U} / \mathrm{L}$

Bilirubin,

umol/L

$9.9(7.1-13.0) ; 216.0 ; \mathrm{n}=12567$

Lipid and

glycemic

profile 
Triglyceride, $\mathrm{mmol} / \mathrm{L}$

Low-density

lipoprotein, $\mathrm{mmol} / \mathrm{L}$

High-density lipoprotein, $\mathrm{mmol} / \mathrm{L}$

Cholesterol, $\mathrm{mmol} / \mathrm{L}$

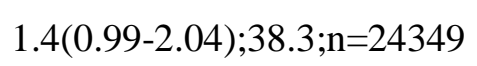

HbA1c, g/dL
Fast glucose,
mmol/L

TyG index

7.32(6.88-7.81);11.61;n=24349

Razard ratio; CI: confidence interval; DM: diabetes mellitus; HF: heart failure; AF: atrial fibrillation; AMI: acute myocardial infarction; VTF: ventricular tachycardia/fibrillation; IHD: ischemic heart disease; COPD: chronic obstructive pulmonary disease; PVD: peripheral vascular disease; TIA: transient ischemic attack; ACEI: angiotensinogen converting enzyme inhibitor; ARB: angiotensin receptor blocker; TyG: triglyceride-glucose
$<0.0001 * *$ $*$

$<0.0001 * *$ $*$

$<0.0001 * *$ $<0.0001 * *$ $*$

$<0.0001 * *$ $<0.0001 * *$
$<0.0001 * *$

acki ACEL angiotensinogenconverting enzyme inhibitor; ARB: angiotenin receptor blocker; TyG: triglyceride-glucose 
Table 2. Cox-proportional hazard model analysis for all-cause mortality, cardiovascular mortality, new onset DM and new onset HF.

$*$ for $\mathrm{p} \leq 0.05, * *$ for $\mathrm{p} \leq 0.01, * * *$ for $\mathrm{p} \leq 0.001$

\begin{tabular}{|c|c|c|c|c|c|c|c|c|}
\hline Characteristics & $\begin{array}{c}\text { All-cause } \\
\text { mortality } \\
\text { HR }[95 \% \text { CI }]\end{array}$ & $P$ value & $\begin{array}{c}\text { Cardiovascular } \\
\text { mortality } \\
\text { HR }[95 \% \mathrm{CI}]\end{array}$ & $P$ value & $\begin{array}{c}\text { New onset DM } \\
\text { HR }[95 \% \text { CI }]\end{array}$ & $P$ value & $\begin{array}{l}\text { New onset HF } \\
\text { HR }[95 \% \mathrm{CI}]\end{array}$ & $P$ value \\
\hline \multicolumn{9}{|l|}{ Demographics } \\
\hline Male gender & $1.44[1.38,1.50]$ & $<0.0001 * * *$ & $1.16[1.03,1.31]$ & $0.0137 *$ & $1.24[1.19,1.30]$ & $<0.0001 * * *$ & $1.28[1.18,1.38]$ & $<0.0001 * * *$ \\
\hline Baseline age, year & $1.11[1.11,1.11]$ & $<0.0001 * * *$ & $1.10[1.09,1.11]$ & $<0.0001 * * *$ & $1.02[1.02,1.02]$ & $<0.0001 * * *$ & $1.08[1.08,1.08]$ & $<0.0001 * * *$ \\
\hline \multicolumn{9}{|l|}{ Past comorbidities } \\
\hline Charlson score & $2.11[2.08,2.13]$ & $<0.0001 * * *$ & $2.07[2.00,2.16]$ & $<0.0001 * * *$ & $1.20[1.18,1.22]$ & $<0.0001 * * *$ & $1.88[1.82,1.93]$ & $<0.0001 * * *$ \\
\hline Liver diseases & $5.09[2.29,11.33]$ & $0.0001 * * *$ & - & - & $2.90[1.09,7.74]$ & $0.0331 *$ & - & - \\
\hline Endocrine & $1.48[0.89,2.46]$ & 0.127 & - & - & $1.38[0.80,2.37]$ & 0.25 & $1.09[0.35,3.37]$ & 0.885 \\
\hline Hypertension & $1.57[1.43,1.71]$ & $<0.0001 * * *$ & $1.64[1.28,2.10]$ & $0.0001 * * *$ & $0.94[0.84,1.05]$ & 0.267 & $1.64[1.39,1.94]$ & $<0.0001 * * *$ \\
\hline IHD & $1.88[1.59,2.22]$ & $<0.0001 * * *$ & $2.78[1.87,4.13]$ & $<0.0001 * * *$ & $1.30[1.06,1.59]$ & $0.0116^{*}$ & $2.53[1.90,3.35]$ & $<0.0001 * * *$ \\
\hline COPD & $5.52[3.83,7.94]$ & $<0.0001 * * *$ & $1.55[0.22,11.00]$ & 0.663 & $1.92[1.12,3.32]$ & $0.0184 *$ & $3.47[1.44,8.34]$ & $0.0055^{* *}$ \\
\hline Gastrointestinal & $1.23[1.08,1.39]$ & $0.0019 * *$ & $0.87[0.57,1.34]$ & 0.529 & $0.95[0.82,1.11]$ & 0.527 & $1.13[0.87,1.46]$ & 0.351 \\
\hline PVD & $4.57[2.60,8.05]$ & $<0.0001 * * *$ & - & - & $2.78[1.39,5.55]$ & $0.0039 * *$ & $4.06[1.31,12.60]$ & $0.0153 *$ \\
\hline TIA/Ischemic stroke & $1.91[1.47,2.48]$ & $<0.0001 * * *$ & $2.51[1.30,4.84]$ & $0.0059 * *$ & $1.14[0.81,1.60]$ & 0.469 & $2.02[1.24,3.31]$ & $0.0050 * *$ \\
\hline Gastrointestinal bleeding & $1.28[0.96,1.70]$ & 0.0895 & $0.43[0.11,1.74]$ & 0.239 & $0.96[0.69,1.34]$ & 0.808 & $1.29[0.75,2.23]$ & 0.354 \\
\hline Cancer & $1.90[1.54,2.34]$ & $<0.0001 * * *$ & $1.04[0.47,2.31]$ & 0.928 & $1.09[0.84,1.43]$ & 0.515 & $1.59[1.02,2.46]$ & $0.0402 *$ \\
\hline \multicolumn{9}{|l|}{ Medications } \\
\hline ACEI & $1.79[1.67,1.91]$ & $<0.0001 * * *$ & $1.92[1.60,2.30]$ & $<0.0001 * * *$ & $2.10[1.97,2.25]$ & $<0.0001 * * *$ & $2.09[1.85,2.35]$ & $<0.0001 * * *$ \\
\hline ARB & $1.56[1.18,2.06]$ & $0.0017 * *$ & $2.06[1.03,4.12]$ & $0.0424 *$ & $2.38[1.83,3.09]$ & $<0.0001 * * *$ & $2.35[1.50,3.69]$ & $0.0002 * * *$ \\
\hline Calcium channel blockers & $1.55[1.46,1.64]$ & $<0.0001 * * *$ & $1.58[1.35,1.86]$ & $<0.0001 * * *$ & $1.54[1.45,1.63]$ & $<0.0001 * * *$ & $1.58[1.42,1.77]$ & $<0.0001 * * *$ \\
\hline Beta blockers & $1.09[1.02,1.16]$ & $0.0113 *$ & $1.20[1.01,1.43]$ & $0.0407 *$ & $1.25[1.18,1.34]$ & $<0.0001 * * *$ & $1.21[1.07,1.36]$ & $0.0019 * *$ \\
\hline Nitrates & $1.83[1.69,1.98]$ & $<0.0001 * * *$ & $2.52[2.06,3.08]$ & $<0.0001 * * *$ & $1.85[1.70,2.01]$ & $<0.0001 * * *$ & $2.28[1.97,2.63]$ & $<0.0001 * * *$ \\
\hline
\end{tabular}


Antihypertensive drugs

Anti-diabetic drugs

Statins and fibrates

Diuretics for hypertension

Anticoagulants

Antiplatelets

Lipid-lowering drugs

\section{Complete cell count}

Mean corpuscular volume, fL $\quad 1.02[1.01,1.02]<0.0001 * * *$ Lymphocyte, $\mathrm{x} 10^{\wedge} 9 / \mathrm{L}$

Metamyelocyte, $\mathrm{x} 10^{\wedge} 9 / \mathrm{L}$

Monocyte, $x 10^{\wedge} 9 / \mathrm{L}$

Neutrophil, $\times 10^{\wedge} 9 / \mathrm{L}$

White cell count, $x 10^{\wedge} 9 / \mathrm{L}$

Mean corpuscular

haemoglobin, pg

Myelocyte, x10^9/L

Platelet, $x 10^{\wedge} 9 / \mathrm{L}$

Reticulocyte, $x 10^{\wedge} 9 / \mathrm{L}$

Red cell count, $x 10^{\wedge} 12 / \mathrm{L}$

Hematocrit, L/L

\section{Liver and renal function tests}

Potassium, mmol/L

Albumin, g/L

Sodium, mmol/L

Urea, mmol/L

Protein, g/L

Creatinine, umol/L

$1.91[1.76,2.07]<0.0001 * * *$

$1.45[1.34,1.56]<0.0001^{* * * *}$ $1.15[1.07,1.23] \quad 0.0002 * * *$ $1.34[1.24,1.45]<0.0001 * * *$ $2.24[1.60,3.13]<0.0001 * * *$ $2.23[2.08,2.39]<0.0001 * * *$ $1.16[1.08,1.26] \quad 0.0002 * * *$ $0.72[0.68,0.76]<0.0001 * * *$ $1.29[0.68,2.42] \quad 0.437$ $2.49[2.23,2.79]<0.0001 * * *$ $1.09[1.08,1.09]<0.0001^{* * *}$ $1.06[1.05,1.06]<0.0001 * * *$ $1.04[1.03,1.05]<0.0001 * * *$ $1.63[0.75,3.55] \quad 0.218$ $1.00[1.00,1.00]<0.0001 * * *$ $1.00[1.00,1.00] \quad 0.545$ $0.62[0.58,0.65]<0.0001 * * *$ $0.01[0.00,0.03]<0.0001 * * *$
$0.98[0.94,1.02] \quad 0.253$ $0.90[0.89,0.91]<0.0001 * * *$ $0.97[0.96,0.97]<0.0001 * * *$ $0.96[0.96,0.97]<0.0001 * * *$ $1.003[1.003$, $1.003]$ $1.12[1.11,1.12]<0.0001 * * *$
$1.58[1.23,2.03]$

$1.43[1.14,1.80]$

$1.61[1.35,1.93]$

$1.29[1.02,1.63]$

$5.98[3.30,10.83]$

$2.68[2.22,3.22]$

$1.63[1.34,1.98]$

$0.0004 * * * \quad 1.60[1.46,1.75]<0.0001 * * * 2.00[1.71,2.34]<0.0001 * * *$ $0.0017 * * \quad 3.17[2.96,3.39]<0.0001 * * * 1.61[1.39,1.86]<0.0001 * * *$ $<0.0001 * * * 1.58[1.47,1.69]<0.0001 * * * 1.15[1.00,1.33] \quad 0.0476 *$ $0.0347 * 1.37[1.26,1.49]<0.0001 * * * 1.41[1.21,1.65]<0.0001 * * *$ $<0.0001 * * * 2.05[1.42,2.95] \quad 0.0001 * * * 2.33[1.21,4.47] \quad 0.0115 *$ $<0.0001 * * * 1.92[1.78,2.08]<0.0001 * * * 2.33[2.03,2.66]<0.0001 * * *$ $<0.0001 * * * 1.53[1.42,1.65]<0.0001 * * * 1.20[1.03,1.40] \quad 0.0173 *$

$1.01[0.99,1.02]$

$0.88[0.74,1.04]$

$2.74[1.97,3.80]$

$1.08[1.05,1.11]$

$1.07[1.05,1.08]$

$1.02[0.99,1.05]$

$1.00[1.00,1.00]$

$1.00[0.99,1.01]$

$0.69[0.59,0.82]$

$0.02[0.00,3.95]$

$0.3681 .01[1.01,1.02]<0.0001 * * * 1.01[1.00,1.02] \quad 0.0140 *$ $0.1420 .95[0.90,1.00] \quad 0.0343 * 0.76[0.68,0.85]<0.0001 * * *$ $0.48[0.18,1.28] \quad 0.142 \quad 0.78[0.20,2.99] \quad 0.712$

$<0.0001 * * * 1.61[1.42,1.83]<0.0001 * * * 2.04[1.60,2.60]<0.0001 * * *$ $<0.0001 * * * 1.05[1.04,1.06]<0.0001 * * * 1.07[1.05,1.09]<0.0001 * * *$ $<0.0001 * * * 1.04[1.04,1.05]<0.0001 * * * 1.05[1.04,1.07]<0.0001 * * *$

$0.2831 .05[1.03,1.06]<0.0001 * * * 1.03[1.01,1.05] \quad 0.0112 *$

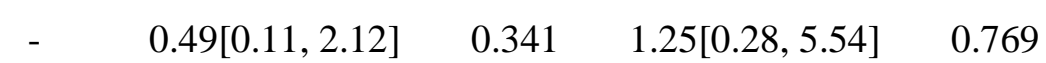

$\begin{array}{lllll}0.51 & 1.00[1.00,1.00] & 0.0001 * * * & 1.00[1.00,1.00] & 0.0002 * * *\end{array}$ $0.717 \quad 1.00[1.00,1.01] \quad 0.0012 * * \quad 1.00[1.00,1.01] \quad 0.625$ $<0.0001 * * * \quad 1.03[0.98,1.09] \quad 0.183 \quad 0.68[0.61,0.75]<0.0001 * * *$ $\begin{array}{lllll}0.149 & 36.01[5.5,236.1] & 0.0002 * * * & 0.09[0.00,4.02] & 0.215\end{array}$

$0.91[0.81,1.02]$ $0.93[0.91,0.95]$ $0.97[0.95,0.98]$ $1.12[1.11,1.14]$ $0.98[0.96,0.99]$

\subsection{4} $<0.0001 * * * 0.97[0.97,0.98]<0.0001 * * * 0.93[0.92,0.94]<0.0001 * * *$ $0.0003 * * * 0.98[0.97,0.98]<0.0001 * * * 0.98[0.97,0.99] \quad 0.0015 * *$ $<0.0001 * * * 1.05[1.04,1.06]<0.0001 * * * 1.12[1.11,1.13]<0.0001 * * *$ $0.0087 * * 0.99[0.98,0.99] \quad 0.0001 * * * 0.96[0.95,0.97]<0.0001 * * *$ $1.002[1.001$

$<0.0001 * * * \quad \begin{gathered}1.002[1.001 \\ 1.002]\end{gathered}$
$<0.0001 * * * \quad 1.003[1.003,1.004]$ 


\begin{tabular}{|c|c|c|c|c|c|c|c|c|}
\hline Alkaline phosphatase, U/L & $\begin{array}{c}1.004[1.004, \\
1.005]\end{array}$ & $<0.0001 * * *$ & $1.004[1.002,1.005]$ & $<0.0001 * * *$ & $\begin{array}{c}1.003[1.002, \\
1.004]\end{array}$ & $<0.0001 * * *$ & $\begin{array}{c}1.003[1.002, \\
1.004]\end{array}$ & $<0.0001 * * *$ \\
\hline Aspartate transaminase, $\mathrm{U} / \mathrm{L}$ & $\begin{array}{c}1.001[1.000 \\
1.001]\end{array}$ & $0.0001 * * *$ & $0.997[0.989,1.005]$ & 0.503 & $\begin{array}{c}1.001[1.001 \\
1.001]\end{array}$ & $<0.0001 * * *$ & $\begin{array}{c}1.000[0.999 \\
1.002]\end{array}$ & 0.728 \\
\hline Alanine transaminase, $\mathrm{U} / \mathrm{L}$ & $\begin{array}{c}0.998[0.996 \\
0.999]\end{array}$ & $0.0012 * *$ & $0.990[0.984,0.996]$ & $0.0018 * *$ & $\begin{array}{c}1.002[1.001 \\
1.003]\end{array}$ & $<0.0001 * * *$ & $\begin{array}{c}0.994[0.991 \\
0.998]\end{array}$ & $0.0009 * * *$ \\
\hline Bilirubin, umol/L & $\begin{array}{c}1.006[1.002 \\
1.010]\end{array}$ & $0.0052 * *$ & $1.00[0.98,1.01]$ & 0.569 & $\begin{array}{c}1.005[1.000 \\
1.009]\end{array}$ & $0.0335^{*}$ & $\begin{array}{c}0.996[0.986 \\
1.006]\end{array}$ & 0.396 \\
\hline \multicolumn{9}{|l|}{ Lipid and gracile profile } \\
\hline Triglyceride, $\mathrm{mmol} / \mathrm{L}$ & $0.98[0.96,1.00]$ & $0.0134 *$ & $1.05[1.01,1.08]$ & $0.0078 * *$ & $1.07[1.06,1.08]$ & $<0.0001 * * *$ & $0.97[0.94,1.01]$ & 0.0940 \\
\hline $\begin{array}{l}\text { Low-density lipoprotein, } \\
\mathrm{mmol} / \mathrm{L}\end{array}$ & $0.97[0.94,0.99]$ & $0.0071 * *$ & $0.98[0.92,1.05]$ & 0.643 & $0.94[0.92,0.96]$ & $<0.0001 * * *$ & $0.97[0.92,1.01]$ & 0.135 \\
\hline $\begin{array}{l}\text { High-density lipoprotein, } \\
\mathrm{mmol} / \mathrm{L}\end{array}$ & $0.80[0.76,0.86]$ & $<0.0001 * * *$ & $0.57[0.48,0.68]$ & $<0.0001 * * *$ & $0.62[0.58,0.66]$ & $<0.0001 * * *$ & $0.80[0.71,0.90]$ & $0.0003 * * *$ \\
\hline Cholesterol, $\mathrm{mmol} / \mathrm{L}$ & $0.93[0.91,0.95]$ & $<0.0001 * * *$ & $0.99[0.94,1.05]$ & 0.778 & $0.95[0.94,0.97]$ & $<0.0001 * * *$ & $0.96[0.92,1.00]$ & $0.0289 *$ \\
\hline $\mathrm{HbA} 1 \mathrm{c}, \mathrm{g} / \mathrm{dL}$ & $0.88[0.86,0.89]$ & $<0.0001 * * *$ & $0.88[0.83,0.93]$ & $<0.0001 * * *$ & $1.09[1.06,1.11]$ & $<0.0001 * * *$ & $1.02[1.01,1.12]$ & $<0.0001 * * *$ \\
\hline Fast glucose, mmol/L & $1.04[1.04,1.05]$ & $<0.0001 * * *$ & $1.05[1.04,1.07]$ & $<0.0001 * * *$ & $1.07[1.07,1.08]$ & $<0.0001 * * *$ & $1.04[1.03,1.05]$ & $<0.0001 * * *$ \\
\hline TyG index & $1.21[1.18,1.25]$ & $<0.0001 * * *$ & $1.56[1.45,1.69]$ & $<0.0001 * * *$ & $1.51[1.47,1.55]$ & $<0.0001 * * *$ & $1.27[1.20,1.34]$ & $<0.0001 * * *$ \\
\hline Decile1 & $0.70[0.65,0.76]$ & $<0.0001 * * *$ & $0.48[0.37,0.63]$ & $<0.0001 * * *$ & $0.55[0.50,0.60]$ & $<0.0001 * * *$ & $0.66[0.56,0.77]$ & $<0.0001 * * *$ \\
\hline Decile2 & $0.88[0.82,0.95]$ & $0.0008 * * *$ & $0.61[0.48,0.78]$ & $0.0001 * * *$ & $0.67[0.61,0.72]$ & $<0.0001 * * *$ & $0.77[0.66,0.89]$ & $0.0004 * * *$ \\
\hline Decile3 & $0.95[0.88,1.02]$ & 0.136 & $0.84[0.68,1.04]$ & 0.103 & $0.69[0.63,0.75]$ & $<0.0001 * * *$ & $0.92[0.80,1.06]$ & 0.25 \\
\hline Decile4 & $0.99[0.92,1.06]$ & 0.748 & $0.92[0.75,1.13]$ & 0.444 & $0.84[0.78,0.90]$ & $<0.0001 * * *$ & $0.97[0.85,1.11]$ & 0.666 \\
\hline Decile5 & $1.03[0.97,1.11]$ & 0.334 & $0.85[0.69,1.05]$ & 0.128 & $1.01[0.94,1.08]$ & 0.811 & $0.96[0.84,1.10]$ & 0.525 \\
\hline Decile6 & $0.93[0.86,0.99]$ & $0.0324 *$ & $0.83[0.67,1.03]$ & 0.0902 . & $1.02[0.95,1.10]$ & 0.533 & $1.05[0.92,1.20]$ & 0.469 \\
\hline Decile7 & $1.06[0.99,1.13]$ & 0.112 & $1.15[0.96,1.38]$ & 0.133 & $1.03[0.96,1.10]$ & 0.414 & $1.11[0.98,1.26]$ & 0.0977 \\
\hline Decile8 & $1.00[0.93,1.07]$ & 0.898 & $1.19[0.99,1.42]$ & 0.0675 . & $1.30[1.22,1.39]$ & $<0.0001 * * *$ & $0.99[0.87,1.14]$ & 0.929 \\
\hline Decile9 & $1.15[1.08,1.23]$ & $<0.0001 * * *$ & $1.44[1.21,1.71]$ & $<0.0001 * * *$ & $1.44[1.35,1.53]$ & $<0.0001 * * *$ & $1.19[1.05,1.35]$ & $0.0059 * *$ \\
\hline Decile10 & $1.36[1.28,1.45]$ & $<0.0001 * * *$ & $1.87[1.60,2.19]$ & $<0.0001 * * *$ & $1.76[1.66,1.87]$ & $<0.0001 * * *$ & $1.44[1.28,1.62]$ & $<0.0001 * * *$ \\
\hline Tertile1 & $0.81[0.77,0.84]$ & $<0.0001 * * *$ & $0.59[0.51,0.67]$ & $<0.0001 * * *$ & $0.57[0.55,0.60]$ & $<0.0001 * * *$ & $0.72[0.66,0.79]$ & $<0.0001 * * *$ \\
\hline
\end{tabular}


Tertile2

$$
1.01[0.97,1.06] \quad 0.659
$$

$0.94[0.83,1.06]$

0.309

$0.98[0.94,1.03]$

0.381

$1.08[0.99,1.17] \quad 0.0760$.

Tertile3

$1.21[1.16,1.27]<0.0001 * * *$

$1.68[1.49,1.88]$

$<0.0001 * * *$

1.67[1.60

acute

: chronic obstructive pulmonary disease; PVD: peripheral vascur disease; TIA: transient ischemic attack; ACEI: angiotensinogen converting enzyme inhibitor; ARB: angiotensin receptor blocker; TyG: triglyceride-glucose

Table 3. Cox-proportional hazard model analysis for new onset AF, AMI, and VTF

$*$ for $\mathrm{p} \leq 0.05, * *$ for $\mathrm{p} \leq 0.01, * * *$ for $\mathrm{p} \leq 0.001$

\begin{tabular}{|c|c|c|c|c|c|c|}
\hline Characteristics & $\begin{array}{l}\text { New onset AF } \\
\text { HR }\left[\begin{array}{ll}95 \% & C I\end{array}\right]\end{array}$ & $P$ value & $\begin{array}{c}\text { New onset AMI } \\
\text { HR }[95 \% \text { CI }]\end{array}$ & $P$ value & $\begin{array}{c}\text { New onset VTF } \\
\text { HR }[95 \% \mathrm{CI}]\end{array}$ & $P$ value \\
\hline \multicolumn{7}{|l|}{ Demographics } \\
\hline Male gender & $1.05[0.97,1.13]$ & 0.2170 & $1.51[1.37,1.65]$ & $<0.0001 * * *$ & $1.75[1.57,1.95]$ & $<0.0001 * * *$ \\
\hline Baseline age, year & $1.09[1.09,1.09]$ & $<0.0001 * * *$ & $1.07[1.07,1.08]$ & $<0.0001 * * *$ & $1.09[1.08,1.10]$ & $<0.0001 * * *$ \\
\hline \multicolumn{7}{|l|}{ Past comorbidities } \\
\hline Charlson score & $1.94[1.89,1.99]$ & $<0.0001 * * *$ & $1.82[1.76,1.88]$ & $<0.0001 * * *$ & $1.97[1.90,2.05]$ & $<0.0001 * * *$ \\
\hline Liver diseases & - & - & - & - & - & - \\
\hline Endocrine & $1.84[0.83,4.11]$ & 0.1350 & $0.47[0.07,3.32]$ & 0.4470 & $0.70[0.10,5.01]$ & 0.7260 \\
\hline Hypertension & $1.48[1.26,1.74]$ & $<0.0001 * * *$ & $1.37[1.12,1.69]$ & $0.0026 * *$ & $1.59[1.26,2.01]$ & $0.0001 * * *$ \\
\hline IHD & $2.09[1.57,2.78]$ & $<0.0001 * * *$ & $2.69[1.97,3.68]$ & $<0.0001 * * *$ & $1.72[1.08,2.74]$ & $0.0224 *$ \\
\hline COPD & $3.16[1.42,7.04]$ & $0.0049 * *$ & $3.61[1.35,9.64]$ & $0.0103 *$ & - & - \\
\hline Gastrointestinal & $1.13[0.90,1.43]$ & 0.2920 & $1.62[1.42,1.91]$ & $0.0141^{*}$ & $1.14[0.80,1.62]$ & 0.4610 \\
\hline PVD & $2.33[0.58,9.32]$ & 0.2320 & $5.82[1.87,18.06]$ & $0.0023 * *$ & $8.09[2.61,25.13]$ & $0.0003 * * *$ \\
\hline TIA/Ischemic stroke & $1.61[0.97,2.67]$ & 0.0664 & $2.13[1.23,3.67]$ & $0.0068 * *$ & $2.68[1.48,4.86]$ & $0.0011 * *$ \\
\hline Gastrointestinal bleeding & $1.98[1.31,2.98]$ & $0.0011 * *$ & $0.62[0.26,1.49]$ & 0.2870 & $1.49[0.75,2.99]$ & 0.2580 \\
\hline Cancer & $1.23[0.78,1.93]$ & 0.3710 & $1.01[0.54,1.87]$ & 0.9890 & $0.60[0.22,1.59]$ & 0.3020 \\
\hline \multicolumn{7}{|l|}{ Medications } \\
\hline ACEI & $1.71[1.52,1.93]$ & $<0.0001 * * *$ & $2.04[1.78,2.35]$ & $<0.0001 * * *$ & $2.26[1.92,2.65]$ & $<0.0001 * * *$ \\
\hline ARB & $2.21[1.44,3.39]$ & $0.0003^{* * *}$ & $2.07[1.20,3.57]$ & $0.0090^{* *}$ & $2.05[1.06,3.94]$ & $0.0323^{*}$ \\
\hline
\end{tabular}




\begin{tabular}{|c|c|c|c|c|c|c|}
\hline Calcium channel blockers & $1.66[1.50,1.84]$ & $<0.0001 * * *$ & $1.67[1.48,1.89]$ & $<0.0001 * * *$ & $1.55[1.33,1.80]$ & $<0.0001 * * *$ \\
\hline Beta blockers & $1.42[1.28,1.58]$ & $<0.0001 * * *$ & $1.42[1.25,1.62]$ & $<0.0001 * * *$ & $1.20[1.02,1.42]$ & $0.0281 *$ \\
\hline Nitrates & $2.76[2.44,3.13]$ & $<0.0001 * * *$ & $2.77[2.38,3.22]$ & $<0.0001 * * *$ & $2.03[1.65,2.50]$ & $<0.0001 * * *$ \\
\hline Antihypertensive drugs & $1.84[1.58,2.13]$ & $<0.0001 * * *$ & $1.74[1.44,2.10]$ & $<0.0001 * * *$ & $2.12[1.72,2.62]$ & $<0.0001 * * *$ \\
\hline Anti-diabetic drugs & $1.01[0.86,1.19]$ & 0.9270 & $1.71[1.45,2.01]$ & $<0.0001 * * *$ & $1.56[1.27,1.91]$ & $<0.0001 * * *$ \\
\hline Statins and fibrates & $1.04[0.91,1.19]$ & 0.5720 & $1.36[1.17,1.58]$ & $<0.0001 * * *$ & $1.24[1.03,1.49]$ & $0.0254 *$ \\
\hline Diuretics for hypertension & $1.48[1.29,1.70]$ & $<0.0001 * * *$ & $1.29[1.08,1.55]$ & $0.0058 * *$ & $1.29[1.03,1.60]$ & $0.0251 *$ \\
\hline Anticoagulants & $11.84[8.48,16.53]$ & $<0.0001 * * *$ & $2.25[1.07,4.72]$ & $0.0324 *$ & $2.91[1.31,6.50]$ & $0.0090 * *$ \\
\hline Antiplatelets & $2.69[2.39,3.02]$ & $<0.0001 * * *$ & $2.64[2.28,3.05]$ & $<0.0001 * * *$ & $2.36[1.96,2.84]$ & $<0.0001 * * *$ \\
\hline Lipid-lowering drugs & $1.10[0.95,1.27]$ & 0.1990 & $1.24[1.04,1.47]$ & $0.0141 *$ & $1.28[1.04,1.57]$ & $0.0179 *$ \\
\hline \multicolumn{7}{|l|}{ Complete cell count } \\
\hline Mean corpuscular volume, fL & $1.014[1.006,1.022]$ & $0.0008 * * *$ & $1.01[1.00,1.02]$ & $0.0054 * *$ & $1.02[1.01,1.04]$ & $0.0002 * * *$ \\
\hline Lymphocyte, x10^9/L & $0.79[0.72,0.88]$ & $<0.0001 * * *$ & $0.87[0.77,0.98]$ & $0.0263 *$ & $0.76[0.65,0.89]$ & $0.0005 * * *$ \\
\hline Metamyelocyte, x10^9/L & $1.03[0.24,4.49]$ & 0.9670 & $0.67[0.05,9.19]$ & 0.7640 & $0.17[0.01,5.72]$ & 0.3270 \\
\hline Monocyte, x10^9/L & $2.15[1.73,2.68]$ & $<0.0001 * * *$ & $2.41[1.86,3.11]$ & $<0.0001 * * *$ & $1.46[0.99,2.17]$ & 0.0586. \\
\hline Neutrophil, x10^9/L & $1.06[1.04,1.08]$ & $<0.0001 * * *$ & $1.09[1.07,1.11]$ & $<0.0001 * * *$ & $1.05[1.02,1.08]$ & $0.0033 * *$ \\
\hline White cell count, $x 10^{\wedge} 9 / \mathrm{L}$ & $1.04[1.02,1.05]$ & $<0.0001 * * *$ & $1.06[1.05,1.07]$ & $<0.0001 * * *$ & $1.04[1.02,1.07]$ & $0.0006 * * *$ \\
\hline Mean cell haemoglobin, pg & $1.04[1.02,1.06]$ & $0.0006 * * *$ & $1.03[1.01,1.06]$ & $0.0080 * *$ & $1.06[1.03,1.09]$ & $0.0004 * * *$ \\
\hline Myelocyte, x10^9/L & $0.15[0.00,12.13]$ & 0.4000 & $1.73[0.03,96.26]$ & 0.7890 & $1.14[0.06,22.93]$ & 0.9340 \\
\hline Platelet, $\times 10^{\wedge} 9 / \mathrm{L}$ & $0.996[0.995,0.997]$ & $<0.0001 * * *$ & $1.00[1.00,1.00]$ & $0.0014 * *$ & $0.997[0.996,0.998]$ & $<0.0001 * * *$ \\
\hline Reticulocyte, x10^9/L & $1.003[0.997,1.009]$ & 0.3430 & $1.01[1.00,1.01]$ & $0.0287 *$ & $1.003[0.995,1.011]$ & 0.5100 \\
\hline Red cell count, $x 10^{\wedge} 12 / \mathrm{L}$ & $0.79[0.71,0.87]$ & $<0.0001 * * *$ & $0.76[0.68,0.86]$ & $<0.0001 * * *$ & $0.72[0.62,0.84]$ & $<0.0001 * * *$ \\
\hline Hematocrit, L/L & $0.04[0.00,1.01]$ & 0.0508 & $0.03[0.00,1.19]$ & 0.0612 . & $0.01[0.00,0.68]$ & $0.0341 *$ \\
\hline \multicolumn{7}{|l|}{ Liver and renal tests } \\
\hline $\mathrm{K} /$ Potassium, mmol/L & $0.83[0.78,0.90]$ & $<0.0001 * * *$ & $0.98[0.90,1.07]$ & 0.7020 & $1.05[0.95,1.17]$ & 0.3470 \\
\hline Albumin, g/L & $0.93[0.91,0.94]$ & $<0.0001 * * *$ & $0.93[0.92,0.95]$ & $<0.0001 * * *$ & $0.94[0.93,0.96]$ & $<0.0001 * * *$ \\
\hline $\mathrm{Na} /$ Sodium, mmol/L & $0.99[0.98,1.01]$ & 0.3890 & $0.97[0.96,0.99]$ & $0.0001 * * *$ & $0.97[0.96,0.99]$ & $0.0016 * *$ \\
\hline Urea, mmol/L & $1.10[1.09,1.11]$ & $<0.0001 * * *$ & $1.12[1.10,1.13]$ & $<0.0001 * * *$ & $1.12[1.11,1.13]$ & $<0.0001 * * *$ \\
\hline Protein, g/L & $0.97[0.96,0.98]$ & $<0.0001 * * *$ & $0.97[0.96,0.98]$ & $<0.0001 * * *$ & $0.96[0.95,0.98]$ & $<0.0001 * * *$ \\
\hline
\end{tabular}




\begin{tabular}{|c|c|c|c|c|c|c|}
\hline Creatinine, umol/L & $1.003[1.002,1.003]$ & $<0.0001 * * *$ & $1.003[1.003,1.004]$ & $<0.0001 * * *$ & $1.003[1.003,1.004]$ & $<0.0001 * * *$ \\
\hline Alkaline phosphatase, U/L & $1.004[1.003,1.005]$ & $<0.0001 * * *$ & $1.003[1.002,1.005]$ & $<0.0001 * * *$ & $1.003[1.001,1.005]$ & $0.0002 * * *$ \\
\hline Aspartate transaminase, $\mathrm{U} / \mathrm{L}$ & $1.000[0.999,1.001]$ & 0.6670 & $1.001[1.000,1.001]$ & 0.1470 & $1.001[0.999,1.002]$ & 0.4260 \\
\hline Alanine transaminase, $\mathrm{U} / \mathrm{L}$ & $0.998[0.995,1.000]$ & 0.0744 & $0.998[0.995,1.001]$ & 0.1990 & $0.991[0.986,0.997]$ & $0.0012 * *$ \\
\hline Bilirubin, umol/L & $1.02[1.01,1.02]$ & $<0.0001 * * *$ & $0.998[0.986,1.009]$ & 0.6780 & $1.00[0.98,1.01]$ & 0.5220 \\
\hline \multicolumn{7}{|l|}{ Lipid and gracile profile } \\
\hline Triglyceride, $\mathrm{mmol} / \mathrm{L}$ & $1.01[1.00,1.04]$ & $<0.0001 * * *$ & $1.03[1.00,1.06]$ & 0.0636 & $0.96[0.92,1.01]$ & 0.1130 \\
\hline Low-density lipoprotein, mmol/L & $0.91[0.87,0.95]$ & $<0.0001 * * *$ & $1.02[0.97,1.08]$ & 0.4070 & $1.00[0.94,1.07]$ & 0.9480 \\
\hline High-density lipoprotein, $\mathrm{mmol} / \mathrm{L}$ & $1.00[0.89,1.11]$ & 0.9340 & $0.49[0.42,0.56]$ & $<0.0001 * * *$ & $0.73[0.61,0.86]$ & $0.0002 * * *$ \\
\hline Cholesterol, mmol/L & $0.90[0.87,0.93]$ & $<0.0001 * * *$ & $1.01[0.97,1.06]$ & 0.5240 & $0.95[0.90,1.00]$ & $0.0493 *$ \\
\hline $\mathrm{HbA} 1 \mathrm{c}, \mathrm{g} / \mathrm{dL}$ & $1.03[1.01,1.05]$ & $0.0001 * * *$ & $1.05[1.01,1.09]$ & $0.0131 *$ & $1.05[1.01,1.06]$ & $0.0379 *$ \\
\hline Fast glucose, mmol/L & $1.02[1.01,1.03]$ & $0.0008 * * *$ & $1.06[1.05,1.07]$ & $<0.0001 * * *$ & $1.04[1.03,1.05]$ & $<0.0001 * * *$ \\
\hline TyG index & $2.36[2.26,2.46]$ & $<0.0001 * * *$ & $1.51[1.42,1.60]$ & $<0.0001 * * *$ & $1.22[1.13,1.31]$ & $<0.0001 * * *$ \\
\hline Decile1 & $0.17[0.13,0.22]$ & $<0.0001 * * *$ & $0.40[0.32,0.50]$ & $<0.0001 * * *$ & $0.66[0.53,0.81]$ & $0.0001 * * *$ \\
\hline Decile2 & $0.25[0.20,0.32]$ & $<0.0001 * * *$ & $0.79[0.67,0.93]$ & $0.0045^{* *}$ & $0.90[0.74,1.09]$ & 0.2630 \\
\hline Decile3 & $0.41[0.35,0.49]$ & $<0.0001 * * *$ & $0.83[0.70,0.97]$ & $0.0216^{*}$ & $0.92[0.76,1.11]$ & 0.3850 \\
\hline Decile4 & $0.49[0.42,0.58]$ & $<0.0001 * * *$ & $0.89[0.76,1.05]$ & 0.1600 & $1.04[0.87,1.25]$ & 0.6460 \\
\hline Decile5 & $0.72[0.63,0.83]$ & $<0.0001 * * *$ & $0.76[0.64,0.90]$ & $0.0015 * *$ & $0.91[0.76,1.10]$ & 0.3530 \\
\hline Decile6 & $0.75[0.66,0.87]$ & $0.0001 * * *$ & $0.96[0.82,1.12]$ & 0.6250 & $1.03[0.86,1.23]$ & 0.7800 \\
\hline Decile7 & $1.19[1.07,1.34]$ & $0.0020 * *$ & $1.05[0.91,1.22]$ & 0.5000 & $0.99[0.82,1.18]$ & 0.8950 \\
\hline Decile8 & $1.49[1.34,1.66]$ & $<0.0001 * * *$ & $1.34[1.17,1.53]$ & $<0.0001 * * *$ & $1.17[0.98,1.38]$ & 0.0805 . \\
\hline Decile9 & $2.22[2.02,2.44]$ & $<0.0001 * * *$ & $1.40[1.22,1.60]$ & $<0.0001 * * *$ & $1.07[0.89,1.28]$ & 0.4570 \\
\hline Decile10 & $3.41[3.13,3.71]$ & $<0.0001 * * *$ & $1.78[1.57,2.02]$ & $<0.0001 * * *$ & $1.37[1.16,1.62]$ & $0.0002 * * *$ \\
\hline Tertile1 & $0.23[0.20,0.26]$ & $<0.0001 * * *$ & $0.61[0.55,0.68]$ & $<0.0001 * * *$ & $0.78[0.69,0.89]$ & $0.0001 * * *$ \\
\hline Tertile2 & $0.73[0.68,1.01]$ & 0.0512 & $0.91[0.82,1.01]$ & 0.0582 & $1.02[0.91,1.14]$ & 0.7540 \\
\hline Tertile3 & $3.51[3.25,3.78]$ & $<0.0001 * * *$ & $1.68[1.54,1.84]$ & $<0.0001 * * *$ & $1.23[1.10,1.38]$ & $0.0003 * * *$ \\
\hline
\end{tabular}

HR: Hazard ratio; CI: confidence interval; DM: diabetes mellitus; HF: heart failure; AF: atrial fibrillation; AMI: acute myocardial infarction; VTF: ventricular tachycardia/fibrillation; IHD: ischemic heart disease; COPD: chronic obstructive pulmonary disease; PVD: peripheral vascular disease; TIA: transient ischemic attack; ACEI: angiotensinogen converting enzyme inhibitor; ARB: angiotensin receptor blocker; TyG: triglyceride-glucose 
Table 4. The hazard ratios (HRs) of primary and secondary outcomes according to tertiles of TyG index.

$*$ for $\mathrm{p} \leq 0.05, * *$ for $\mathrm{p} \leq 0.01, * * *$ for $\mathrm{p} \leq 0.001$

HR hazard ratios; IR: incidence rate.

Model 1 adjusted for none.

Model 2 adjusted for significant demographics.

Model 3 adjusted for significant demographics and past comorbidities.

Model 4 adjusted for significant demographics, past comorbidities and medications.

\begin{tabular}{|c|c|c|c|c|c|c|}
\hline \multirow{2}{*}{ Outcomes } & \multicolumn{2}{|l|}{ Model 1} & \multicolumn{3}{|c|}{ Model 2} & \multirow[b]{2}{*}{ TyG $>7.63$} \\
\hline & TyG $<6.98$ & TyG $\in[6.98,7.63]$ & TyG>7.63 & TyG $<6.98$ & TyG $\in[6.98,7.63]$ & \\
\hline All-cause mortality & 1.00 (reference) & $1.01[0.97,1.06]$ & $1.21[1.16,1.27]^{* * *}$ & 1.00 (reference) & $0.96[0.92,1.01]$ & $1.19[1.14,1.24] * * *$ \\
\hline CAD mortality & 1.00 (reference) & $0.94[0.83,1.06]$ & $1.68[1.49,1.88]^{* * *}$ & 1.00 (reference) & $0.89[0.78,1.01]$ & $1.62[1.44,1.82]^{* * *}$ \\
\hline New onset DM & 1.00 (reference) & $0.98[0.94,1.03]$ & $1.67[1.60,1.75]^{* * *}$ & 1.00 (reference) & $0.96[0.92,1.01]$ & $1.63[1.56,1.70]^{* * *}$ \\
\hline New onset HF & 1.00 (reference) & $1.08[0.99,1.17]$ & $1.25[1.16,1.36]^{* * *}$ & 1.00 (reference) & $1.03[0.95,1.12]$ & $1.21[1.11,1.31]^{* * * *}$ \\
\hline New onset AF & 1.00 (reference) & $0.73[0.68,1.01]$ & $3.51[3.25,3.78]^{* * *}$ & 1.00 (reference) & $0.7[0.64,1.01]$ & $3.48[3.23,3.76]^{* * *}$ \\
\hline New onset AMI & 1.00 (reference) & $0.91[0.82,1.01]$ & $1.68[1.54,1.84] * * *$ & 1.00 (reference) & $0.87[0.79,1.01]$ & $1.62[1.48,1.78]^{* * *}$ \\
\hline New onset VTF & 1.00 (reference) & $1.02[0.91,1.14]$ & $1.23[1.10,1.38]^{* * *}$ & 1.00 (reference) & $0.97[0.87,1.09]$ & $1.2[1.07,1.35]^{* * *}$ \\
\hline \multirow{2}{*}{ Outcomes } & Model 3 & \multicolumn{5}{|c|}{ Model 4} \\
\hline & TyG $<6.98$ & $T y G \in[6.98,7.63]$ & TyG $>7.63$ & TyG $<6.98$ & TyG $\in[6.98,7.63]$ & TyG $>7.63$ \\
\hline All-cause mortality & 1.00 (reference) & $0.96[0.92,1.01]$ & $1.19[1.14,1.24] * * *$ & 1.00 (reference) & $0.96[0.92,1.01]$ & $1.17[1.12,1.22] * * *$ \\
\hline CAD mortality & 1.00 (reference) & $0.89[0.79,1.01]$ & $1.62[1.44,1.82] * * *$ & 1.00 (reference) & $0.89[0.79,1.03]$ & $1.59[1.41,1.79]^{* * *}$ \\
\hline New onset DM & 1.00 (reference) & $0.96[0.92,1.01]$ & $1.63[1.56,1.7] * * *$ & 1.00 (reference) & $0.98[0.93,1.02]$ & $1.52[1.46,1.59]^{* * * *}$ \\
\hline New onset HF & 1.00 (reference) & $1.03[0.95,1.12]$ & $1.21[1.12,1.31]^{* * *}$ & 1.00 (reference) & $1.03[0.95,1.12]$ & $1.19[1.09,1.29]^{* * *}$ \\
\hline New onset AF & 1.00 (reference) & $0.7[0.64,1.01]$ & $3.49[3.23,3.76]^{* * *}$ & 1.00 (reference) & $0.68[0.63,1.04]$ & $3.61[3.34,3.89]^{* * * *}$ \\
\hline New onset AMI & 1.00 (reference) & $0.87[0.79,1.01]$ & $1.63[1.48,1.78]^{* * *}$ & 1.00 (reference) & $0.87[0.79,1.06]$ & $1.59[1.45,1.74]^{* * * *}$ \\
\hline New onset VTF & 1.00 (reference) & $0.97[0.86,1.09]$ & $1.2[1.08,1.35]^{* *}$ & 1.00 (reference) & $0.97[0.86,1.09]$ & $1.18[1.06,1.33]^{* *}$ \\
\hline
\end{tabular}

HR: Hazard ratio; CI: confidence interval; DM: diabetes mellitus; HF: heart failure; AF: atrial fibrillation; AMI: acute myocardial infarction; VTF: ventricular tachycardia/fibrillation; TyG: triglyceride-glucose 
Table 5. Optimal cut-offs between triglyceride level and primary and secondary outcomes with maximal survival rank statistics approach $*$ for $\mathrm{p} \leq 0.05, * *$ for $\mathrm{p} \leq 0.01, * * *$ for $\mathrm{p} \leq 0.001$

\begin{tabular}{|c|c|c|c|c|}
\hline & TyG cutoff value & $\begin{array}{l}<\text { Cut-off value } \\
\text { HR }[95 \% \text { CI }]\end{array}$ & $\begin{array}{l}\geq \text { Cut-off value } \\
\text { HR }[95 \% \text { CI }]\end{array}$ & $P$ for $\log$ ratio test \\
\hline All-cause mortality & 8.05 & 1.0 (reference) & $1.34[1.28,1.42]^{* * *}$ & $<0.0001$ \\
\hline Cardiovascular mortality & 7.43 & 1.0 (reference) & $1.79[1.59,2.02]^{* * *}$ & $<0.0001$ \\
\hline New onset DM & 7.68 & 1.0 (reference) & $1.71[1.64,1.79]^{* * *}$ & $<0.0001$ \\
\hline New onset HF & 7.24 & 1.0 (reference) & $1.35[1.24,1.47]^{* * *}$ & $<0.0001$ \\
\hline New onset AF & 7.75 & 1.0 (reference) & $3.58[3.33,3.86]^{* * *}$ & $<0.0001$ \\
\hline New onset AMI & 7.66 & 1.0 (reference) & $1.75[1.6,1.92]^{* * *}$ & $<0.0001$ \\
\hline New onset VTF & 7.79 & 1.0 (reference) & $1.33[1.18,1.5]^{* * *}$ & $<0.0001$ \\
\hline
\end{tabular}

HR: Hazard ratio; CI: confidence interval; DM: diabetes mellitus; HF: heart failure; AF: atrial fibrillation; AMI: acute myocardial infarction; VTF: ventricular tachycardia/fibrillation; TyG: triglyceride-glucose

Multivariate Cox regression was performed to examine the association between triglyceride levels and primary and secondary outcomes; Effects include significant demographics, past comorbidities, and medications. 


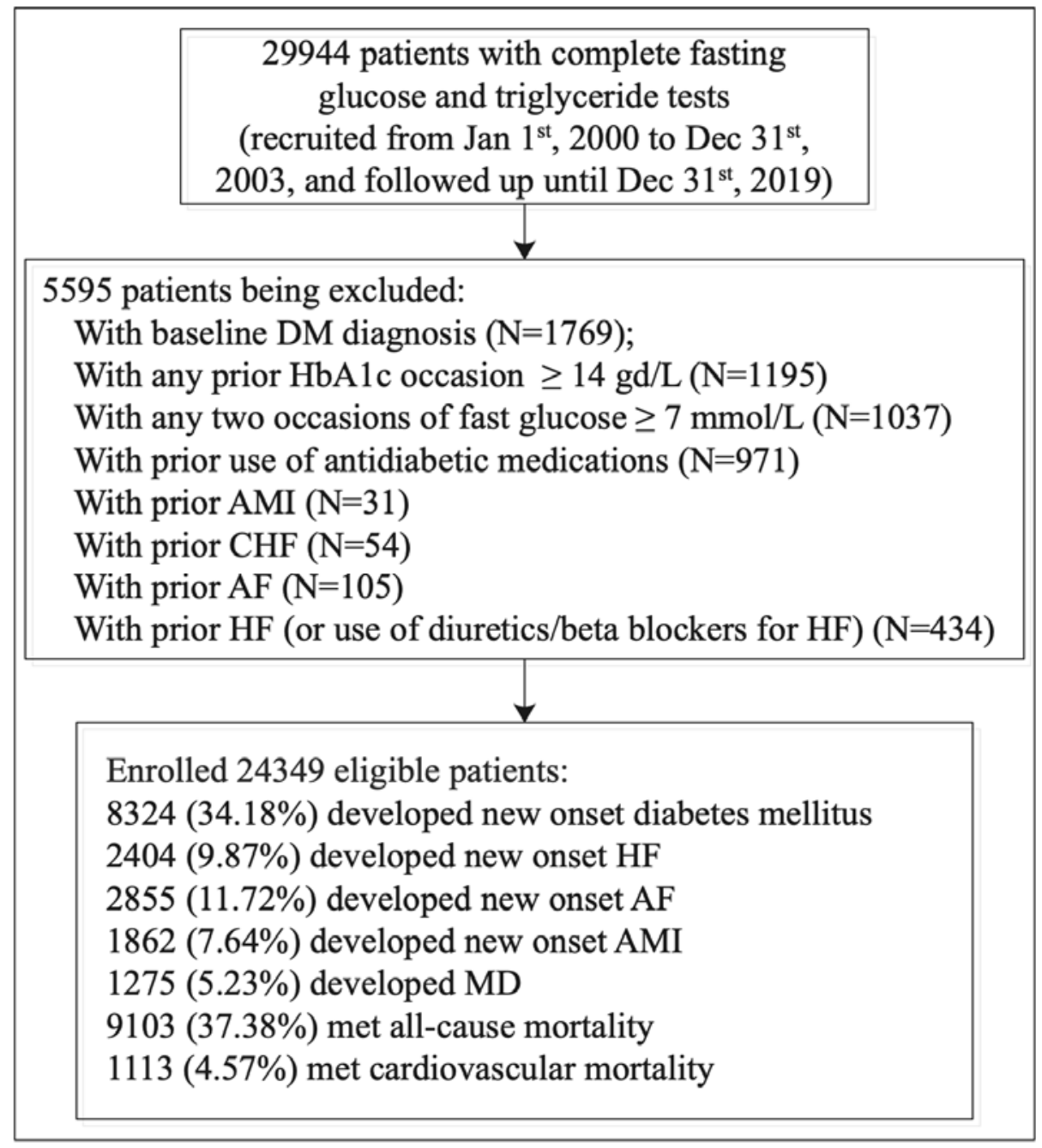

Figure 1. Flow chart of data processing for the study cohort. 
All-cause mortality
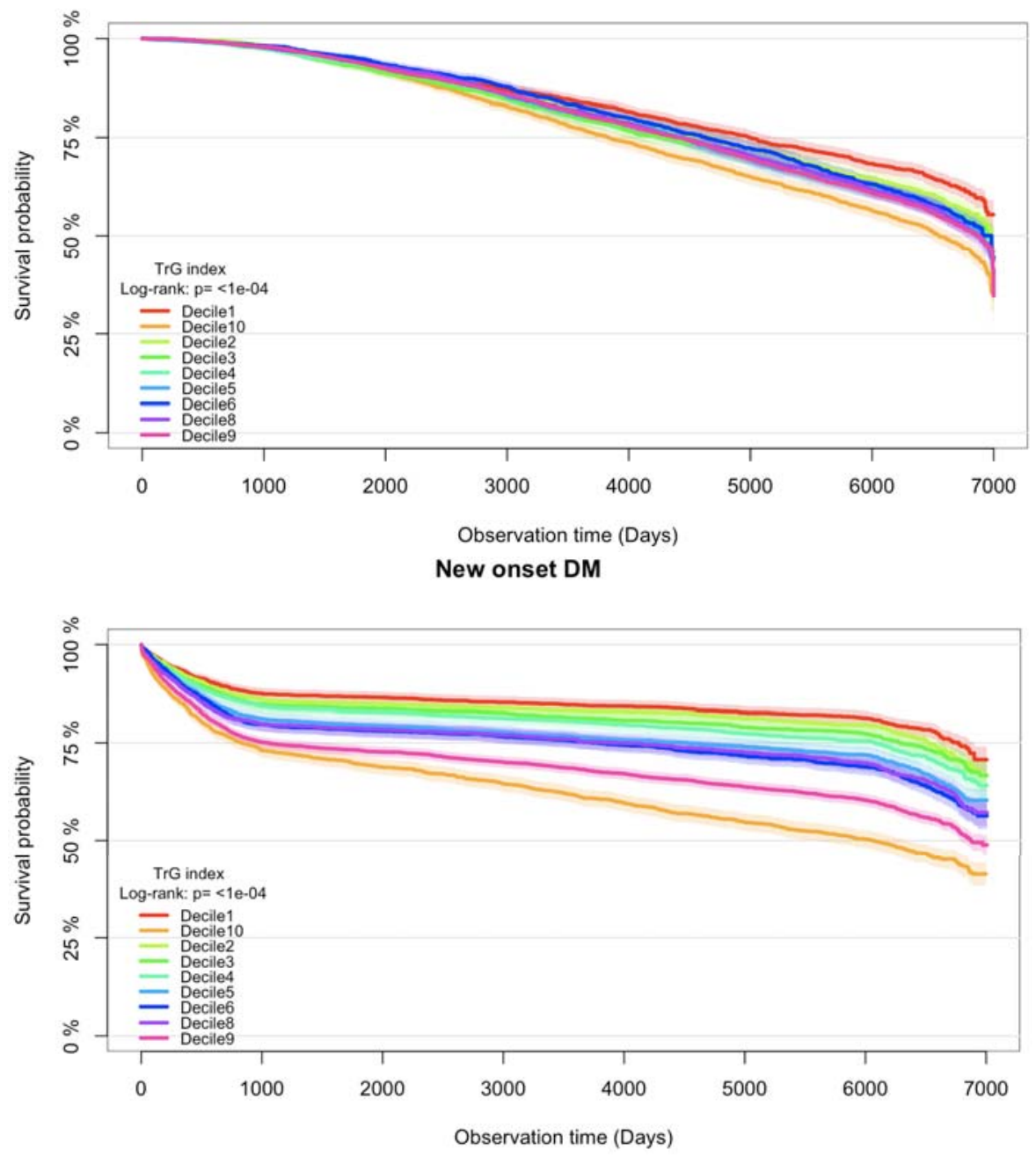

Cardiovascular mortality
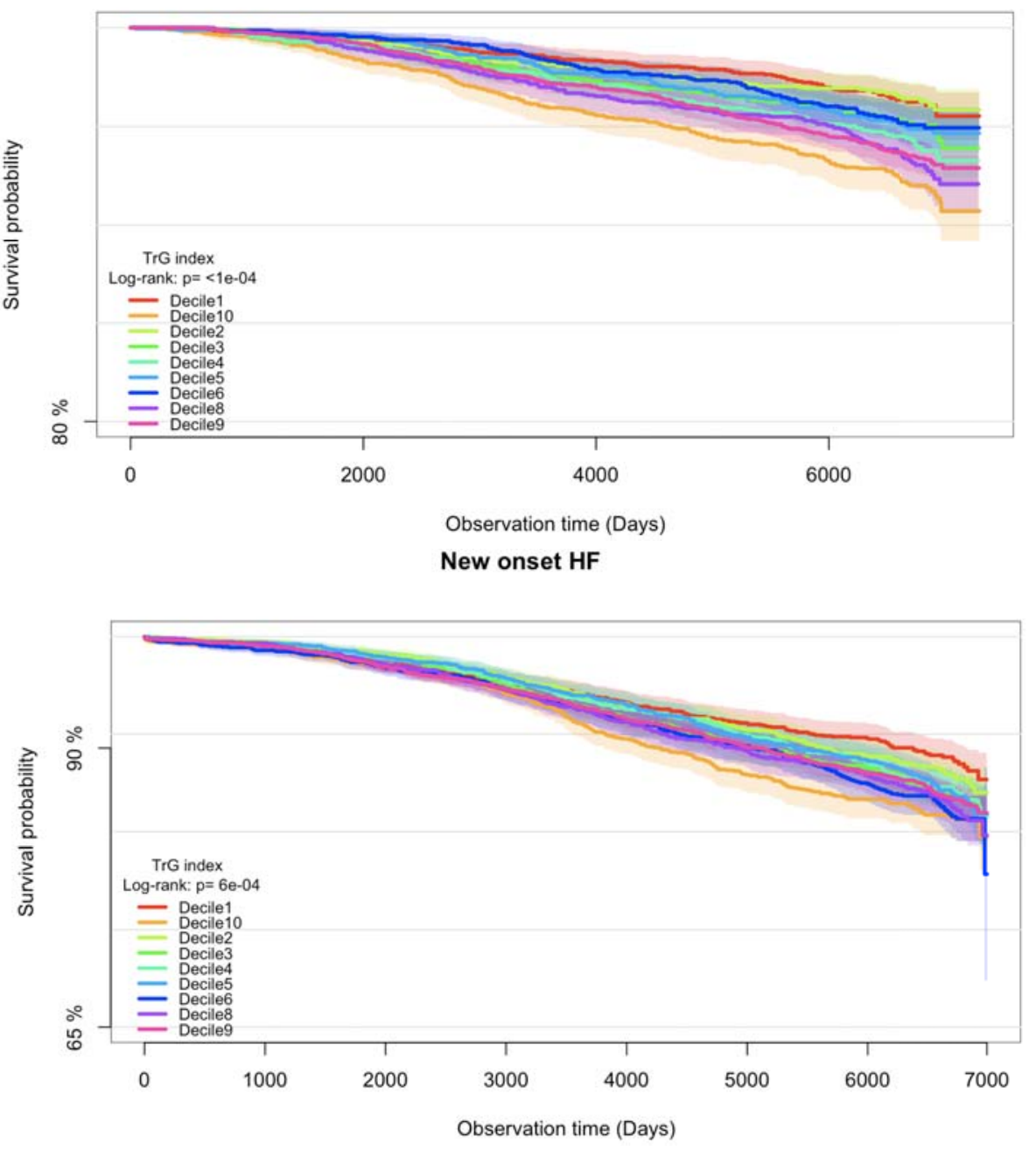

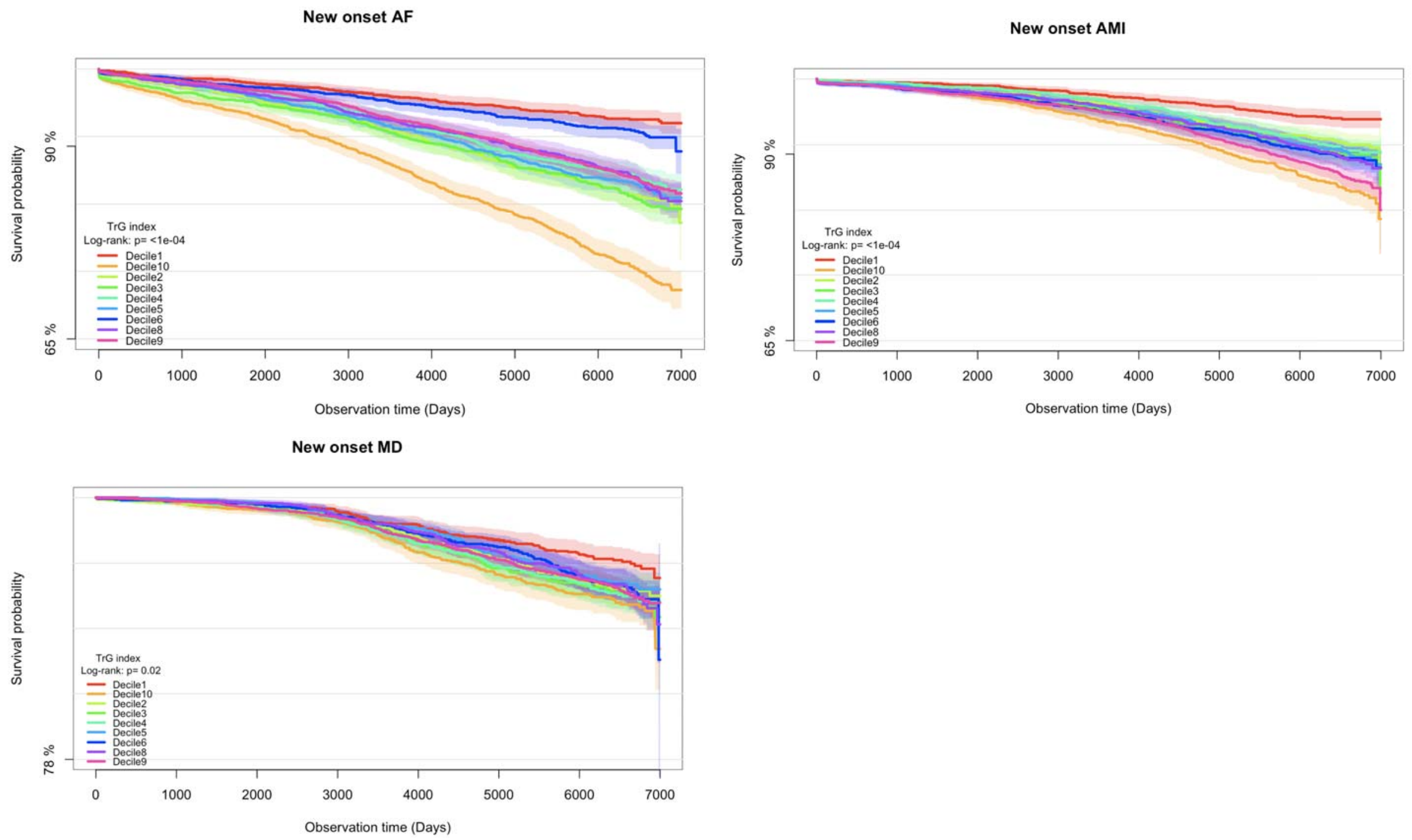

Figure 2. Kaplan-Meier survival curves for primary and secondary outcomes stratified by TyG index deciles. TyG: triglyceride glucose. 
All-cause mortality hazard ratio as a function of TyG index

Adjusted for significant demographics, past comorbidities and medication

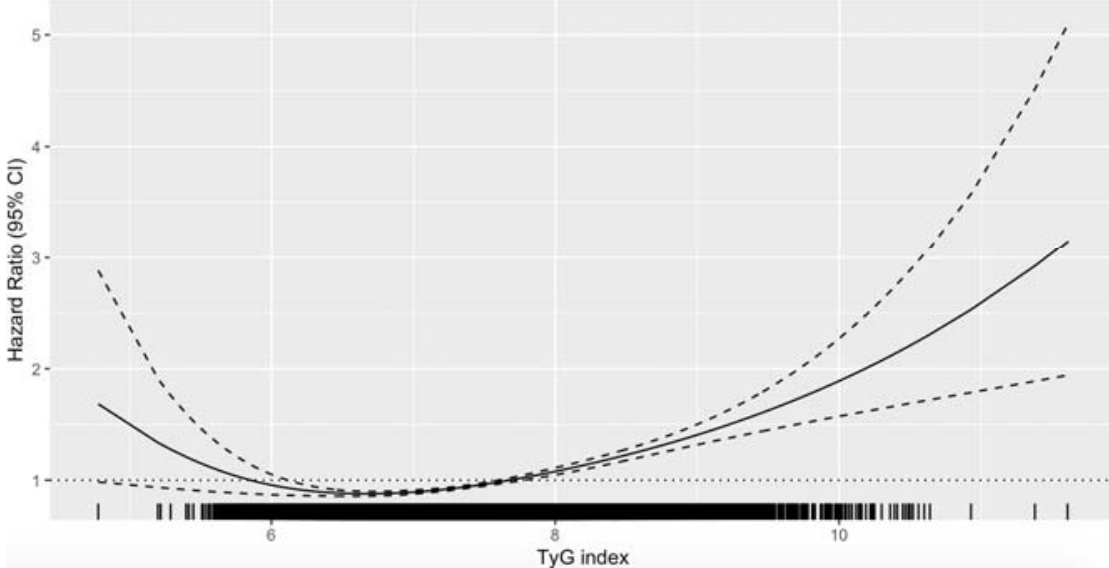

New onset $\mathrm{HF}$ hazard ratio as a function of TyG index

Adjusted for significant demographics, past comorbidities and medications

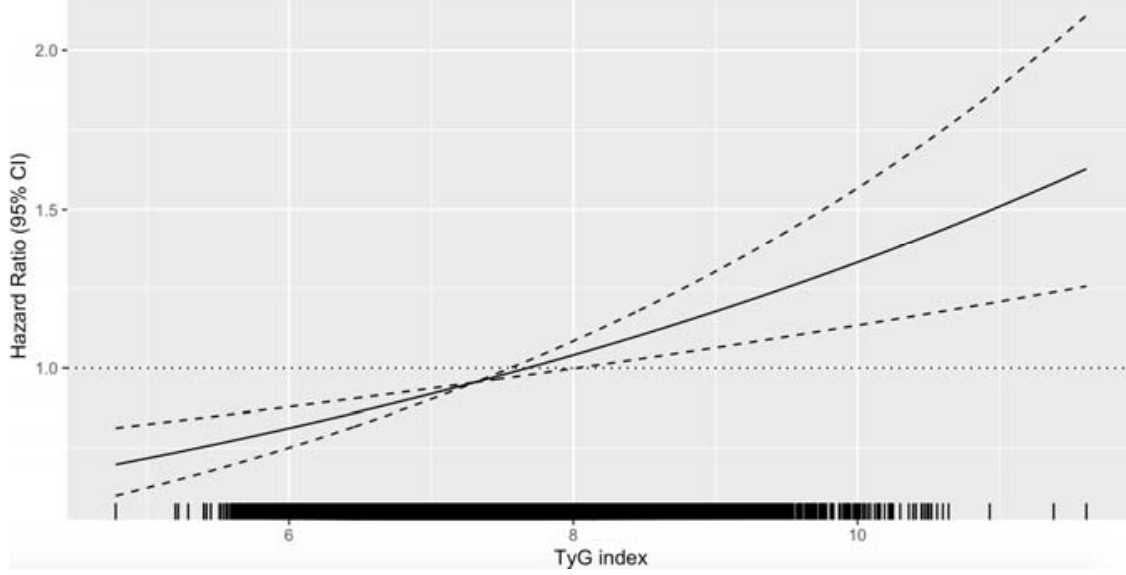

Cardiovascular mortality hazard ratio as a function of TyG index

Adjusted for significant demographics, past comorbidities and medication

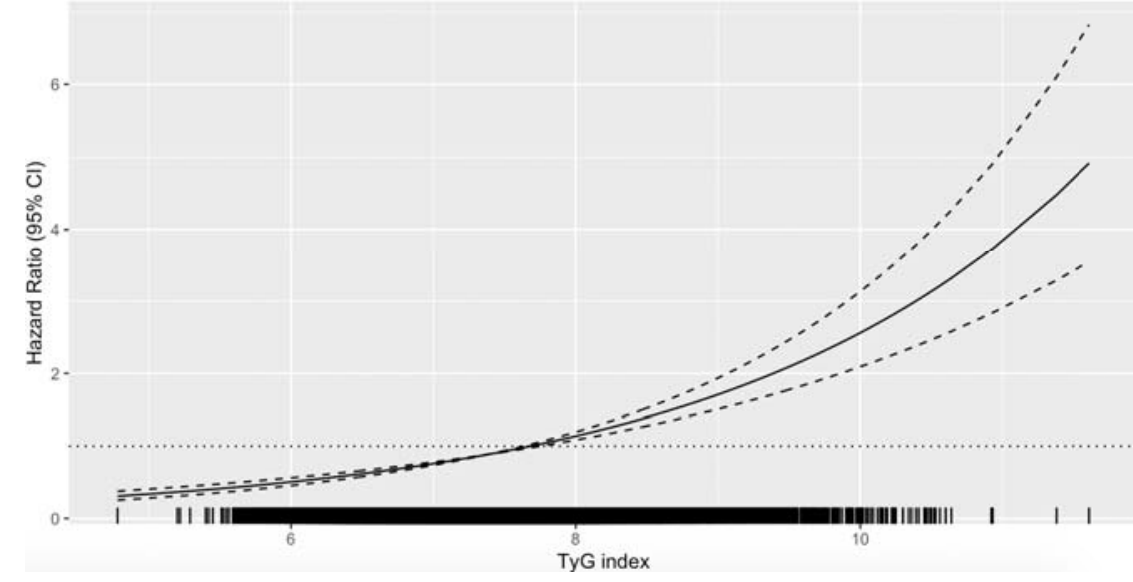

New onset AF hazard ratio as a function of TyG index

Adjusted for significant demographics, past comorbidities and medications

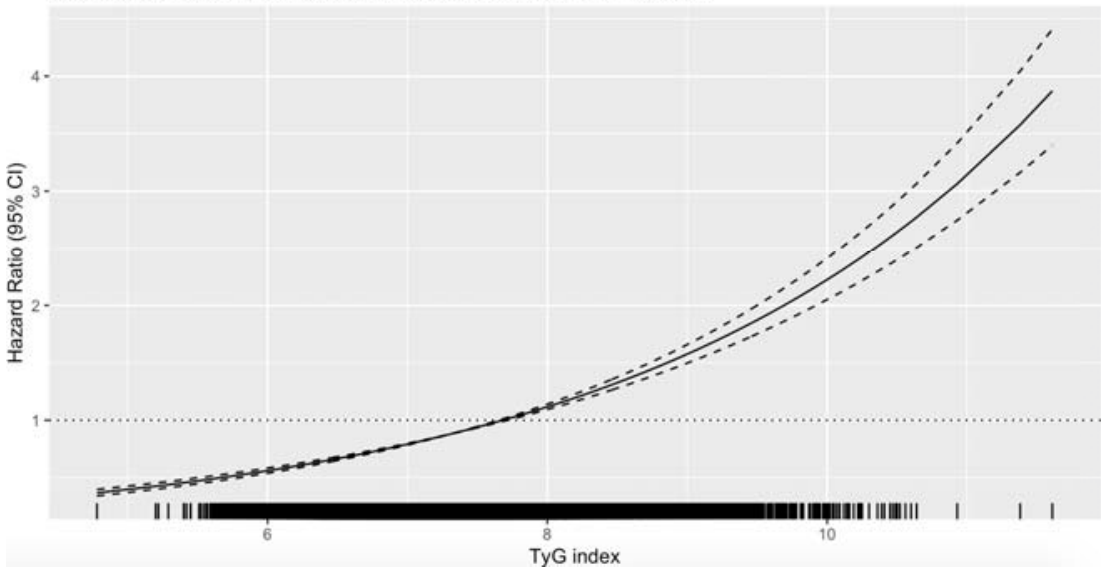



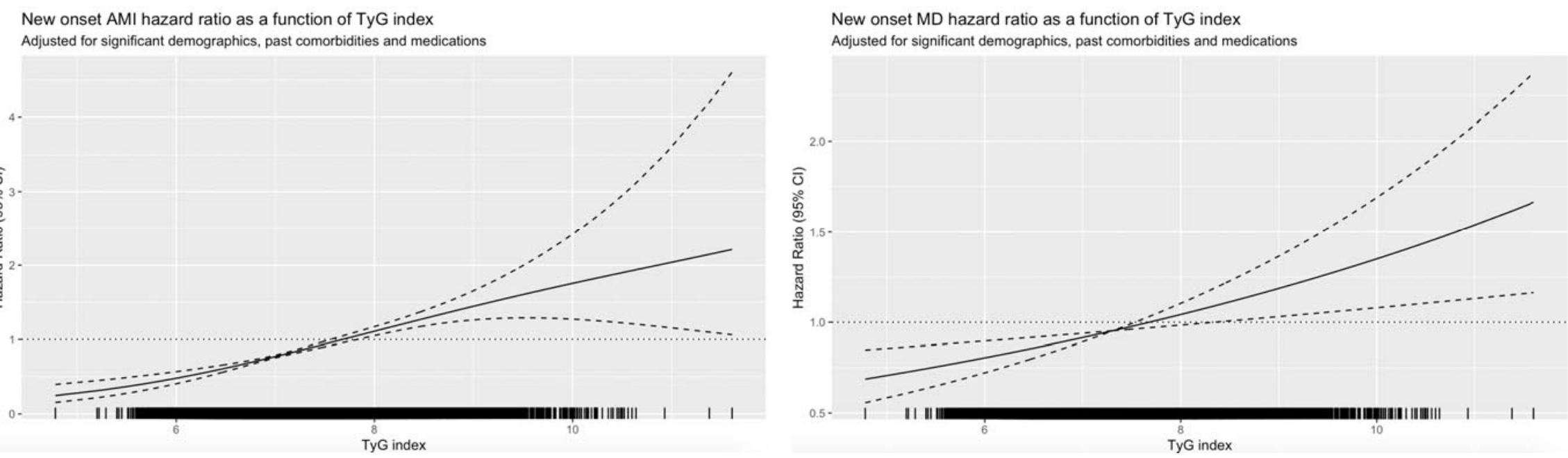

Figure 3. Adjusted cubic spline model of the associations between TyG index and risk of primary and secondary outcomes. TyG: triglyceride glucc 
All-cause mortality

Distribution

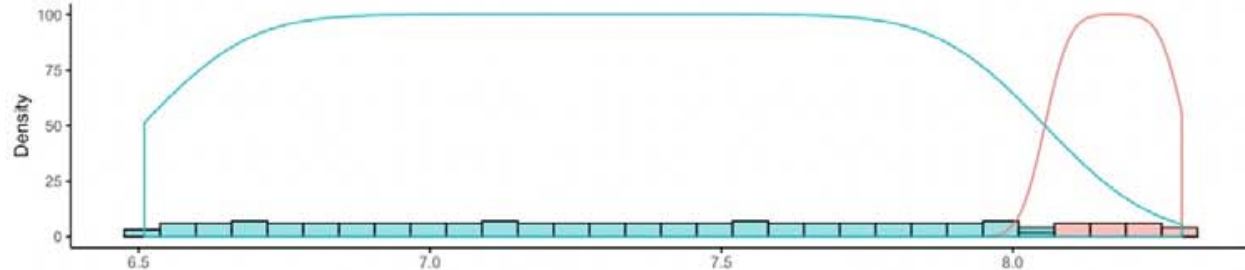

Maximally Selected Rank Statistics

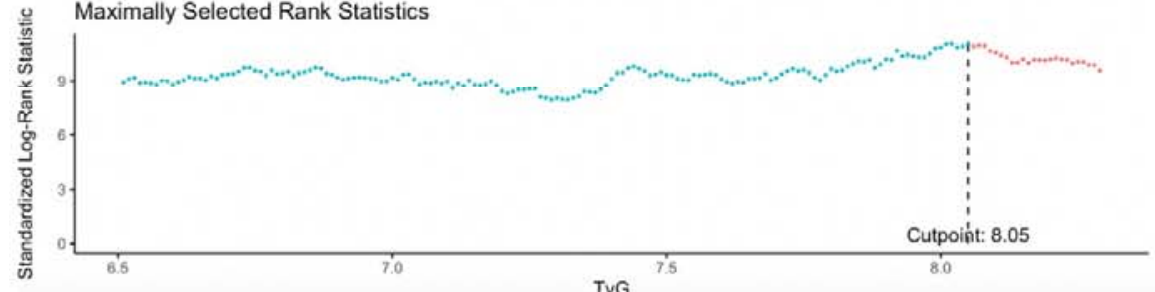

New onset DM

Distribution

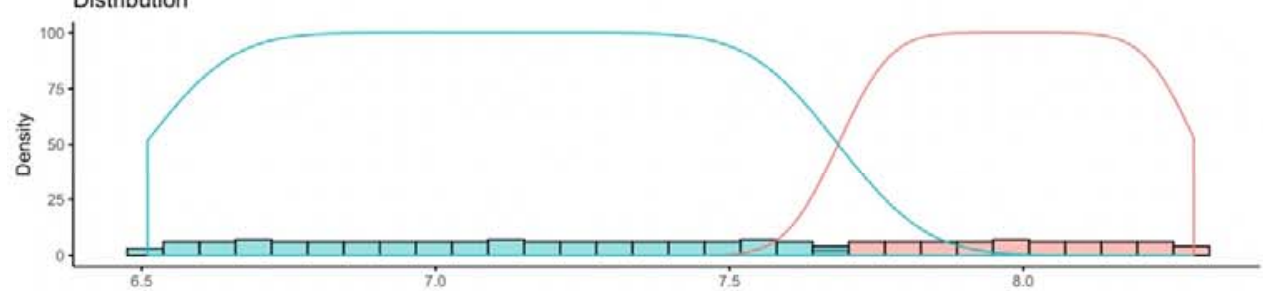

Maximally Selected Rank Statistics

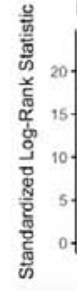

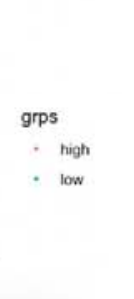

TyG

80
Cardiovascular mortality

Distribution

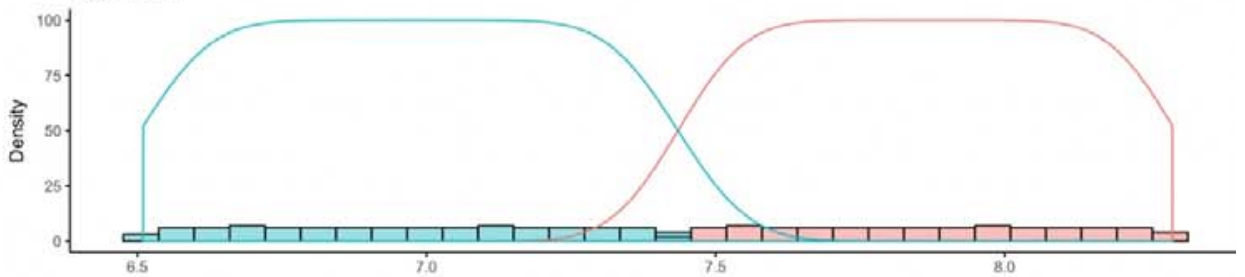

Maximally Selected Rank Statistics

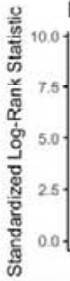

New onset HF

Distribution

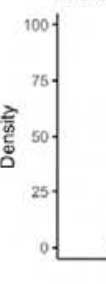

흔.

grps

- low

Cutpoint 7.43

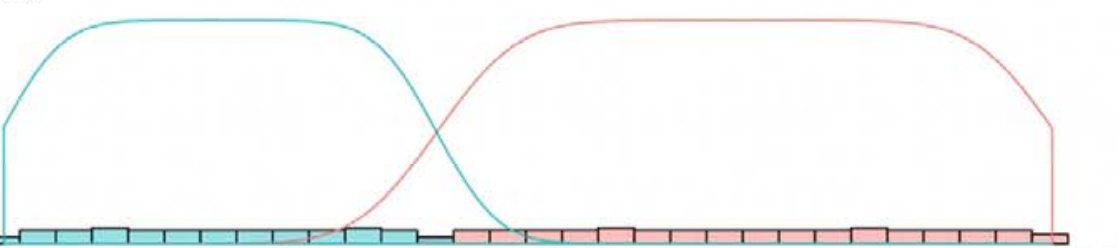

$1+1,10$

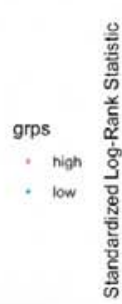

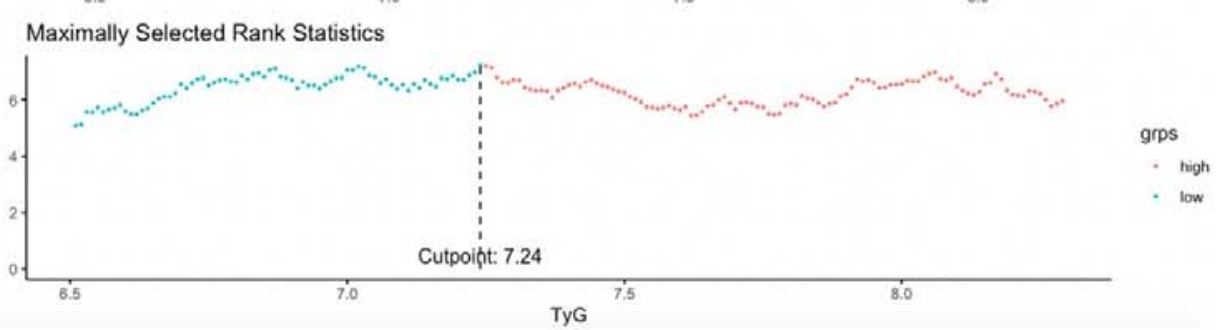

Cutpoint 7.68 


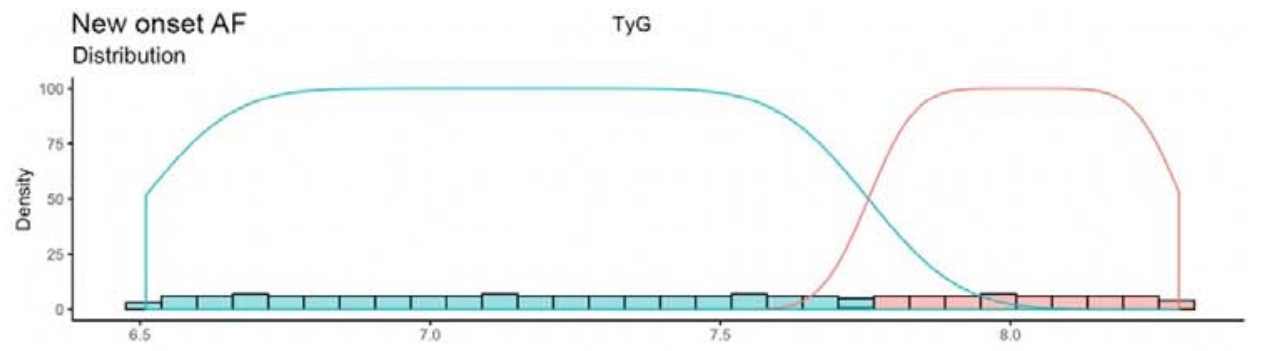

New onset AM
Distribution
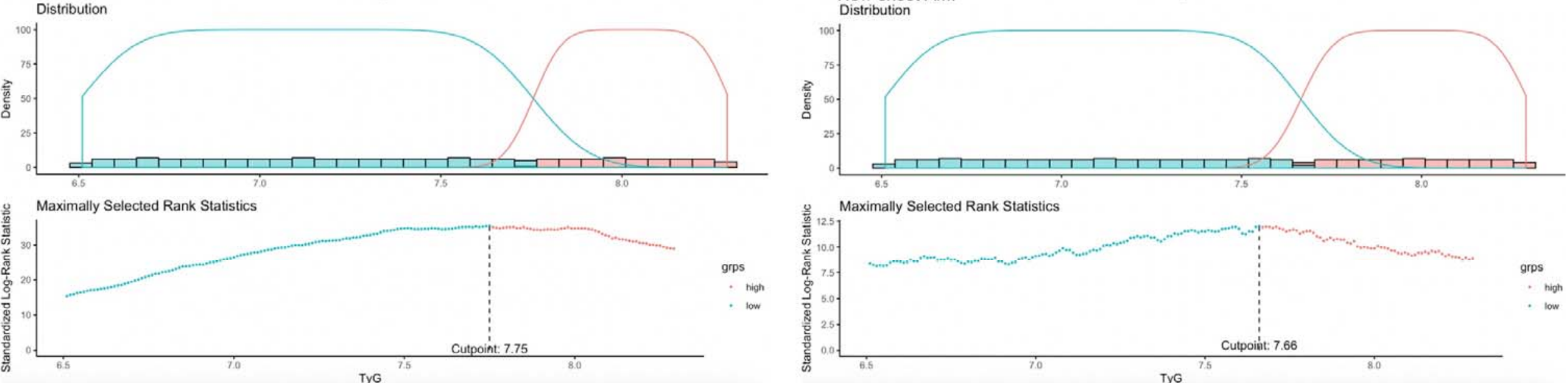

TyG

TyG

New onset MD Distribution

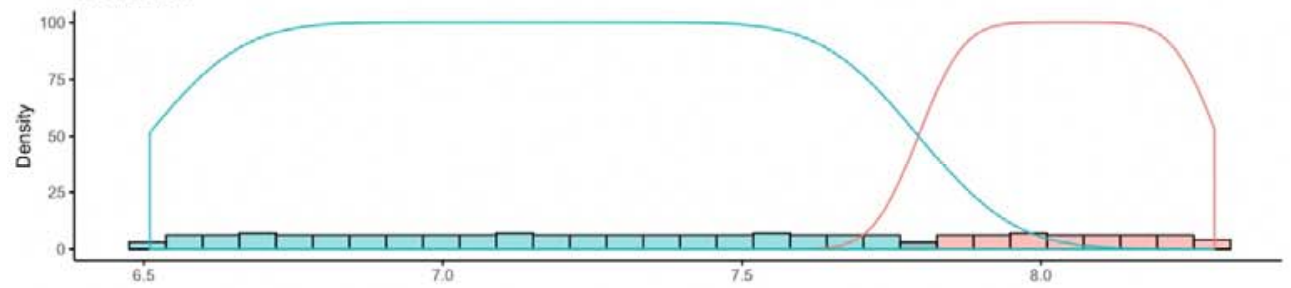

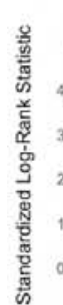

Maximally Selected Rank Statistics

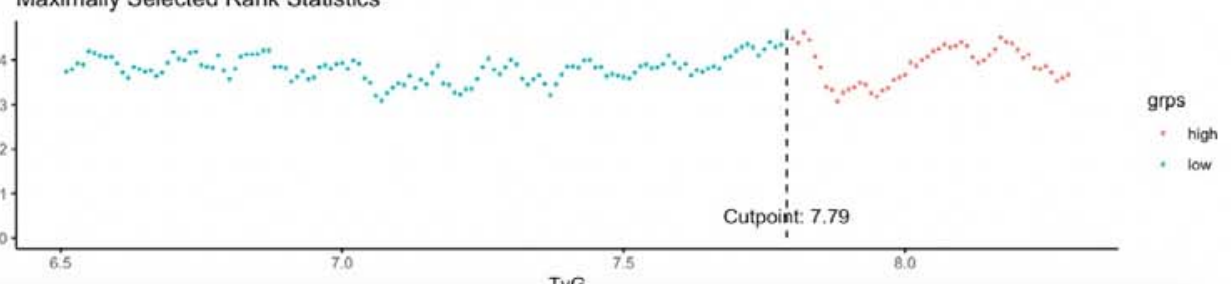

Figure 4. Optimal cutoffs of TyG index to predict primary and secondary outcomes with maximally selected rank statistics approach. 